\title{
Cascade [1,5]-Hydride Transfer/Cyclization for Synthesis of $[3,4]$-Fused Oxindoles
}

Shuai Zhu, ${ }^{\dagger}$ Chunqi Chen, ${ }^{\dagger}$ Kang Duan,${ }^{\dagger}$ Yun-Ming Sun,${ }^{\dagger}$ Shuai-Shuai Li, ${ }^{*}{ }^{\dagger}$ Qing Liu, ${ }^{\S}$ Jian Xiao*,t,

${ }^{\dagger}$ Shandong Province Key Laboratory of Applied Mycology, College of Chemistry and Pharmaceutical Sciences, Qingdao Agricultural University, Qingdao 266109, China

\$College of Marine Science and Engineering, Qingdao Agricultural University, Qingdao, 266109, China.

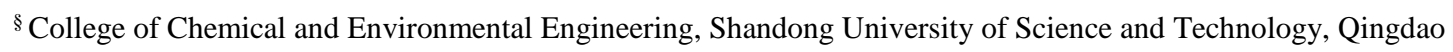
266590, China.

\section{Supporting Information}

1. Optimization of Reaction Conditions for Asymmetric Synthesis...........S2

2. Crystal Structure and Data..............................................S4

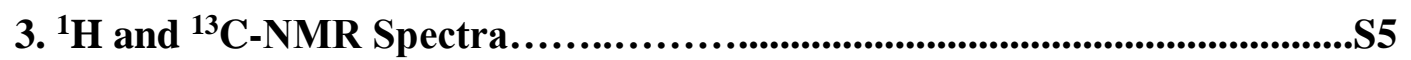


1. Table S1. Optimization of Reaction Conditions for Asymmetric Synthesis

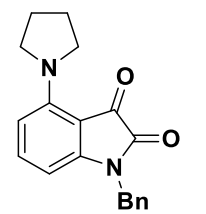

$1 \mathbf{a}$<smiles>c1cc(C2=N[C@H]3Cc4ccccc4[C@H]3O2)nc(C2=N[C@@H]3Cc4ccccc4[C@H]3O2)c1</smiles>

L1<smiles>O=C1CC(=O)c2ccccc21</smiles>

L4<smiles>CCC[18N]C1COC(C(C)(C)C2=NC([PH+])CO2)=N1</smiles>

L7

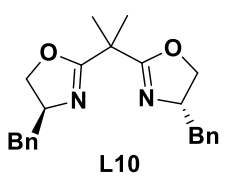<smiles>O=P(O)(O)Oc1c(Cl)cc2ccccc2c1-c1c(O)c(Cl)cc2ccccc12</smiles>

L12

L12a, G=2,6-bis[4-(2-naphthalenyl)phenyl]

L12b, G=2,6-Bis(triphenylsilyl)

L12c, G=2,6-di-2-naphthaleny

L12d, G=2,6-Bis[3,5-bis(trifluoromethyl)phenyl]

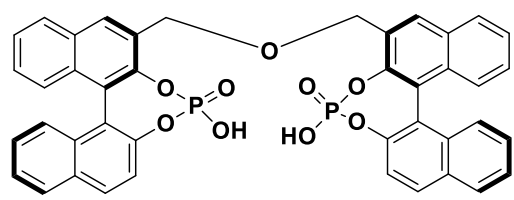

L14

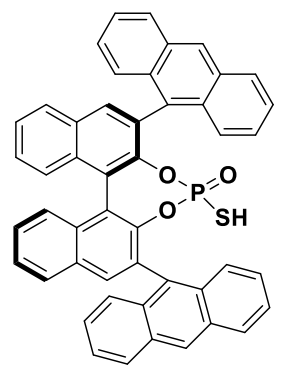

L16<smiles></smiles>

L11 2a

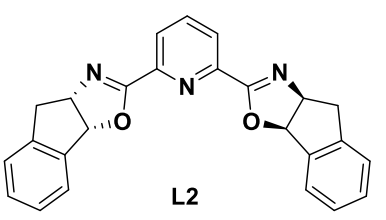<smiles>CC(C)(C)[C@@H]1COC(c2cccc(C3=N[C@H](C(C)(C)C)CO3)n2)=N1</smiles>

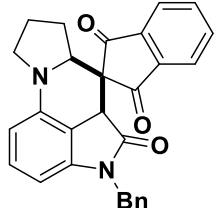

3a
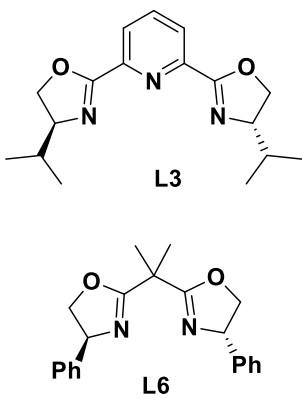

L8

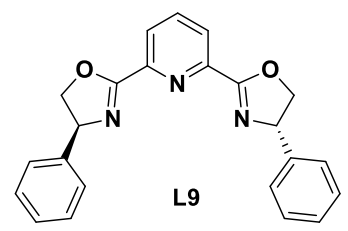

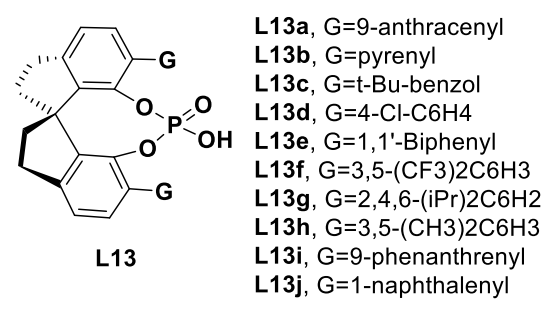

(c)

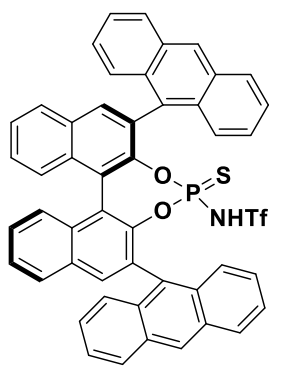

L17 


\begin{tabular}{|c|c|c|c|c|c|c|}
\hline Entry & Cat. $(10 \%)$ & Ligand $(10 \%)$ & Temp. $\left({ }^{\circ} \mathrm{C}\right)$ & Time (h) & Yield/\% & Ee $(\%)$ \\
\hline 1 & $\mathrm{Sc}(\mathrm{OTf})_{3}$ & L1 & 40 & 20 & 90 & 29 \\
\hline 2 & $\mathrm{Sc}(\mathrm{OTf})_{3}$ & L2 & 40 & 20 & 85 & 19 \\
\hline 3 & $\mathrm{Sc}(\mathrm{OTf})_{3}$ & L3 & 40 & 20 & 95 & 19 \\
\hline 4 & $\mathrm{Sc}(\mathrm{OTf})_{3}$ & L4 & 40 & 20 & 80 & 7 \\
\hline 5 & $\mathrm{Sc}(\mathrm{OTf})_{3}$ & L5 & 40 & 20 & 20 & 0 \\
\hline 6 & $\mathrm{Sc}(\mathrm{OTf})_{3}$ & L6 & 40 & 20 & 40 & 6 \\
\hline 7 & $\mathrm{Sc}(\mathrm{OTf})_{3}$ & L7 & 40 & 20 & 0 & 0 \\
\hline 8 & $\mathrm{Sc}(\mathrm{OTf})_{3}$ & L8 & 40 & 20 & 30 & 3 \\
\hline 9 & $\mathrm{Sc}(\mathrm{OTf})_{3}$ & L9 & 40 & 20 & 50 & 10 \\
\hline 10 & $\mathrm{Sc}(\mathrm{OTf})_{3}$ & L10 & 40 & 20 & 70 & 5 \\
\hline 11 & $\mathrm{Sc}(\mathrm{OTf})_{3}$ & L11 & 40 & 20 & 95 & -9 \\
\hline 12 & $\mathrm{Sc}(\mathrm{OTf})_{3}$ & L12a & 60 & 30 & 40 & -9 \\
\hline 13 & $\mathrm{Sc}(\mathrm{OTf})_{3}$ & L12b & 60 & 30 & 50 & 10 \\
\hline 14 & $\mathrm{Sc}(\mathrm{OTf})_{3}$ & $\mathrm{~L} 12 \mathrm{c}$ & 60 & 30 & 51 & 21 \\
\hline 15 & $\mathrm{Sc}(\mathrm{OTf})_{3}$ & L12d & 60 & 30 & 46 & 12 \\
\hline 16 & $\mathrm{Sc}(\mathrm{OTf})_{3}$ & L13a & 60 & 30 & 48 & 30 \\
\hline 17 & $\mathrm{Sc}(\mathrm{OTf})_{3}$ & L13b & 60 & 30 & 40 & 20 \\
\hline 18 & $\mathrm{Sc}(\mathrm{OTf})_{3}$ & $\mathrm{~L} 13 \mathrm{c}$ & 60 & 30 & 44 & 29 \\
\hline 19 & $\mathrm{Sc}(\mathrm{OTf})_{3}$ & L13d & 60 & 30 & 43 & -7 \\
\hline 20 & $\mathrm{Sc}(\mathrm{OTf})_{3}$ & L13e & 60 & 30 & 40 & -2 \\
\hline 21 & $\mathrm{Sc}(\mathrm{OTf})_{3}$ & L13f & 40 & 30 & 58 & 12 \\
\hline 22 & $\mathrm{Sc}(\mathrm{OTf})_{3}$ & L13g & 60 & 30 & 44 & -2 \\
\hline 23 & $\mathrm{Sc}(\mathrm{OTf})_{3}$ & L13h & 60 & 30 & 52 & 18 \\
\hline 24 & $\mathrm{Sc}(\mathrm{OTf})_{3}$ & L13i & 60 & 30 & 60 & 31 \\
\hline 25 & $\mathrm{Sc}(\mathrm{OTf})_{3}$ & $\mathrm{~L} 13 \mathrm{j}$ & 60 & 30 & 50 & -7 \\
\hline 26 & $\mathrm{Sc}(\mathrm{OTf})_{3}$ & L14 & 60 & 30 & 62 & 30 \\
\hline 27 & $\mathrm{Sc}(\mathrm{OTf})_{3}$ & L15 & 60 & 30 & 53 & 30 \\
\hline 28 & $\mathrm{Sc}(\mathrm{OTf})_{3}$ & L16 & 60 & 30 & 48 & -6 \\
\hline 29 & $\mathrm{Sc}(\mathrm{OTf})_{3}$ & L17 & 60 & 30 & 20 & -7 \\
\hline 30 & $\mathrm{Sc}(\mathrm{OTf})_{3}$ & L13a (15\%) & 60 & 30 & 50 & 34 \\
\hline 31 & $\mathrm{Sc}(\mathrm{OTf})_{3}$ & L13a (20\%) & 60 & 30 & 50 & 35 \\
\hline
\end{tabular}

1a $(0.1 \mathrm{mmol}), 2 \mathrm{a}(0.15 \mathrm{mmol}), \mathrm{Sc}(\mathrm{OTf})_{3}(0.01 \mathrm{mmol})$, Ligand (x mol\%), $5 \AA \mathrm{MS}(50 \mathrm{mg})$ in $1.0 \mathrm{~mL}$ distilled DCE at 40 or $60^{\circ} \mathrm{C}$; isolated yield after column chromatography; ee was determined by chiral HPLC.

Figure S1. The ee determined by chiral HPLC.
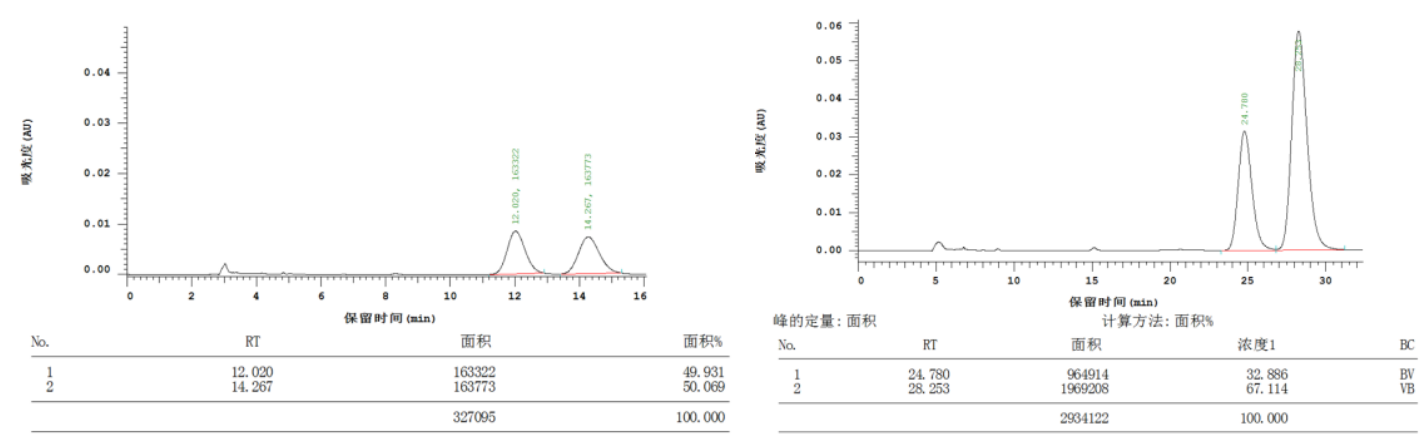


\section{Crystal Structure and Data}

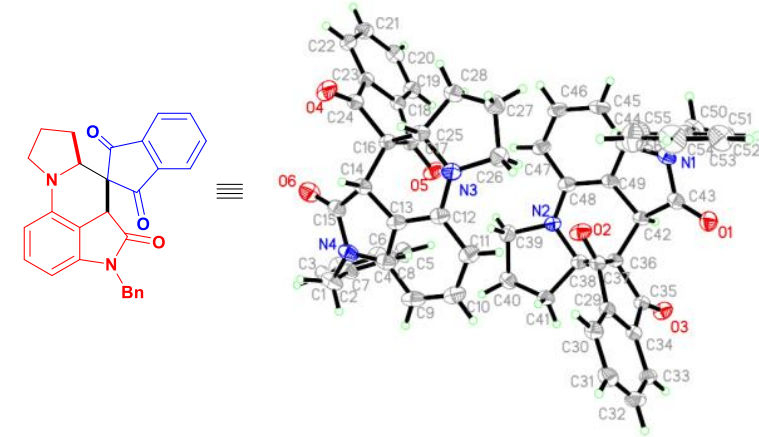

3a (CCDC 1873496)

Figure S2. ORTEP diagram of compound 3a, thermal ellipsoids are drawn on $30 \%$ probability level

Table S2. Crystal data and structure refinement for $3 a$.

Identification code

Empirical formula

Formula weight

Temperature

Wavelength

Crystal system, space group

Unit cell dimensions

Volume

Z, Calculated density

Absorption coefficient

$\mathrm{F}(000)$

Crystal size

Theta range for data collection

Limiting indices

Reflections collected / unique

Completeness to theta $=67.25$

Max. and min. transmission

Refinement method

Data / restraints / parameters

Goodness-of-fit on $\mathrm{F}^{\wedge} 2$

Final $\mathrm{R}$ indices $[\mathrm{I}>2 \operatorname{sigma}(\mathrm{I})]$

$\mathrm{R}$ indices (all data)

Largest diff. peak and hole 3a

$\mathrm{C}_{28} \mathrm{H}_{22} \mathrm{~N}_{2} \mathrm{O}_{3}$

434.16

293(2) K

$1.54184 \mathrm{~A}$

Monoclinic, $\mathrm{C} 2 / \mathrm{c}$

$\mathrm{a}=53.283(2) \mathrm{A} \quad$ alpha $=90 \mathrm{deg}$.

$\mathrm{b}=7.9450(2) \mathrm{A} \quad$ beta $=91.672(4) \mathrm{deg}$.

$\mathrm{c}=20.8497(7) \mathrm{A} \quad$ gamma $=90 \mathrm{deg}$

$8822.6(5) \mathrm{A}^{\wedge} 3$

$8,1.372 \mathrm{Mg} / \mathrm{m}^{\wedge} 3$

$1.257 \mathrm{~mm}^{\wedge}-1$

3816

$0.08 \times 0.07 \times 0.07 \mathrm{~mm}$

3.32 to $67.25 \mathrm{deg}$.

$-63<=\mathrm{h}<=63,-9<=\mathrm{k}<=5,-23<=\mathrm{l}<=24$

$28658 / 7924[\mathrm{R}(\mathrm{int})=0.0379]$

$100.0 \%$

0.9172 and 0.9061

Full-matrix least-squares on $\mathrm{F}^{\wedge} 2$

7924 / 0 / 609

1.039

$\mathrm{R} 1=0.0725, \mathrm{wR} 2=0.2203$

$\mathrm{R} 1=0.0955, \mathrm{wR} 2=0.2434$

0.334 and -1.221 e. $\mathrm{A}^{\wedge}-3$ 


\section{3. ${ }^{1} \mathrm{H}$ and ${ }^{13} \mathrm{C}$-NMR Spectra}

4-benzyl-4,5a,6a,7,8,9-hexahydro-5H-spiro[dipyrrolo[1,2-a:4',3',2'-de]quinoline-6,2'-indene]-1',3', 5-trione (3a)

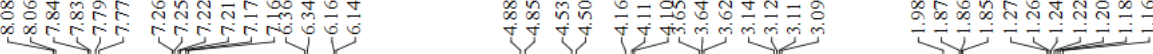
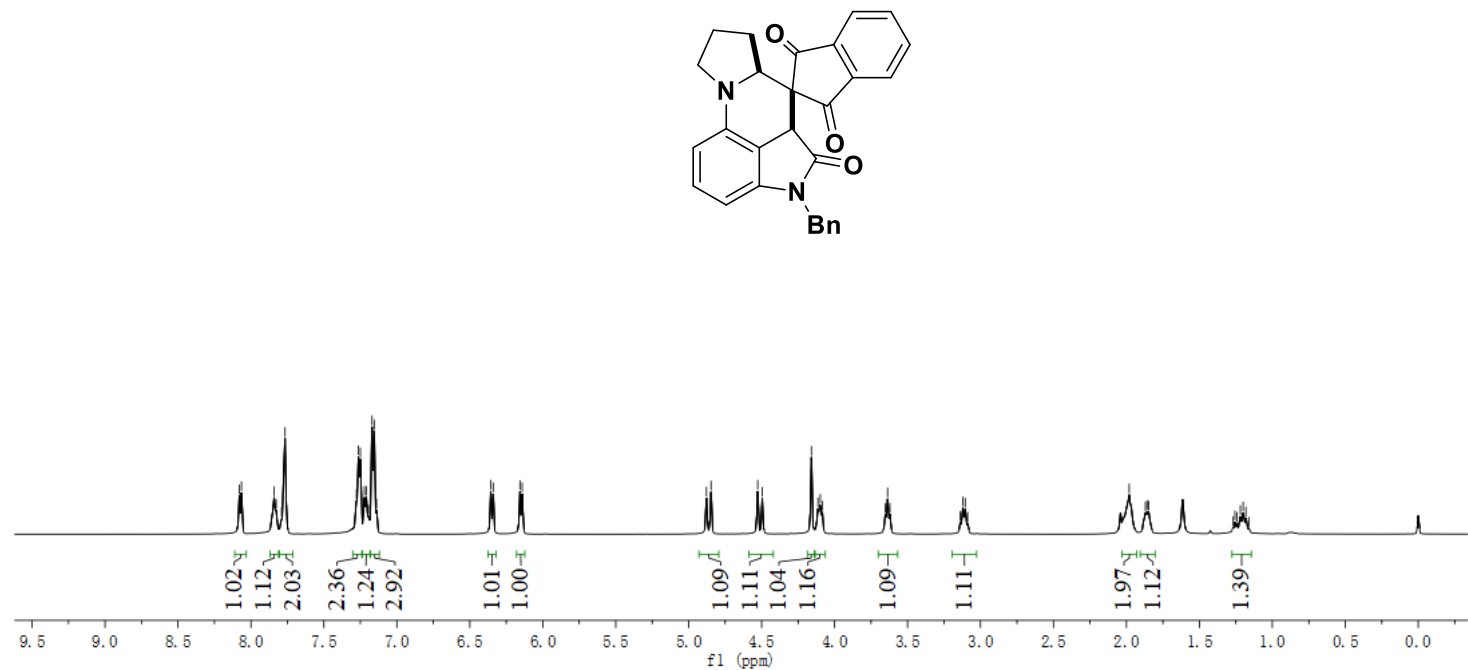

\begin{tabular}{|c|c|c|c|c|}
\hline 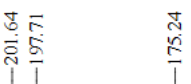 & 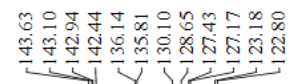 & 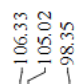 & m & 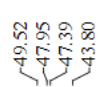 \\
\hline
\end{tabular}
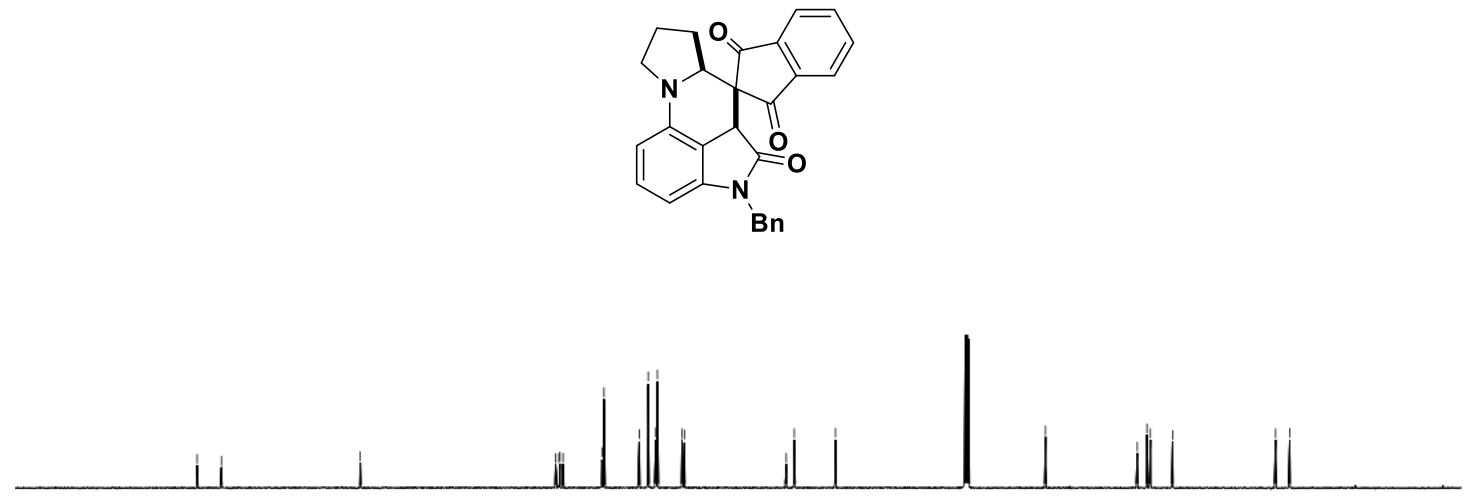

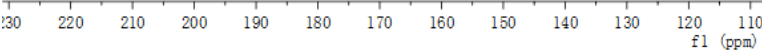


4-(2-chlorobenzyl)-4,5a,6a,7,8,9-hexahydro-5H-spiro[dipyrrolo[1,2-a:4',3',2'-de]quinoline-6,2'-ind ene]-1',3',5-trione (3b)

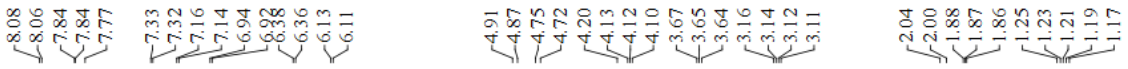
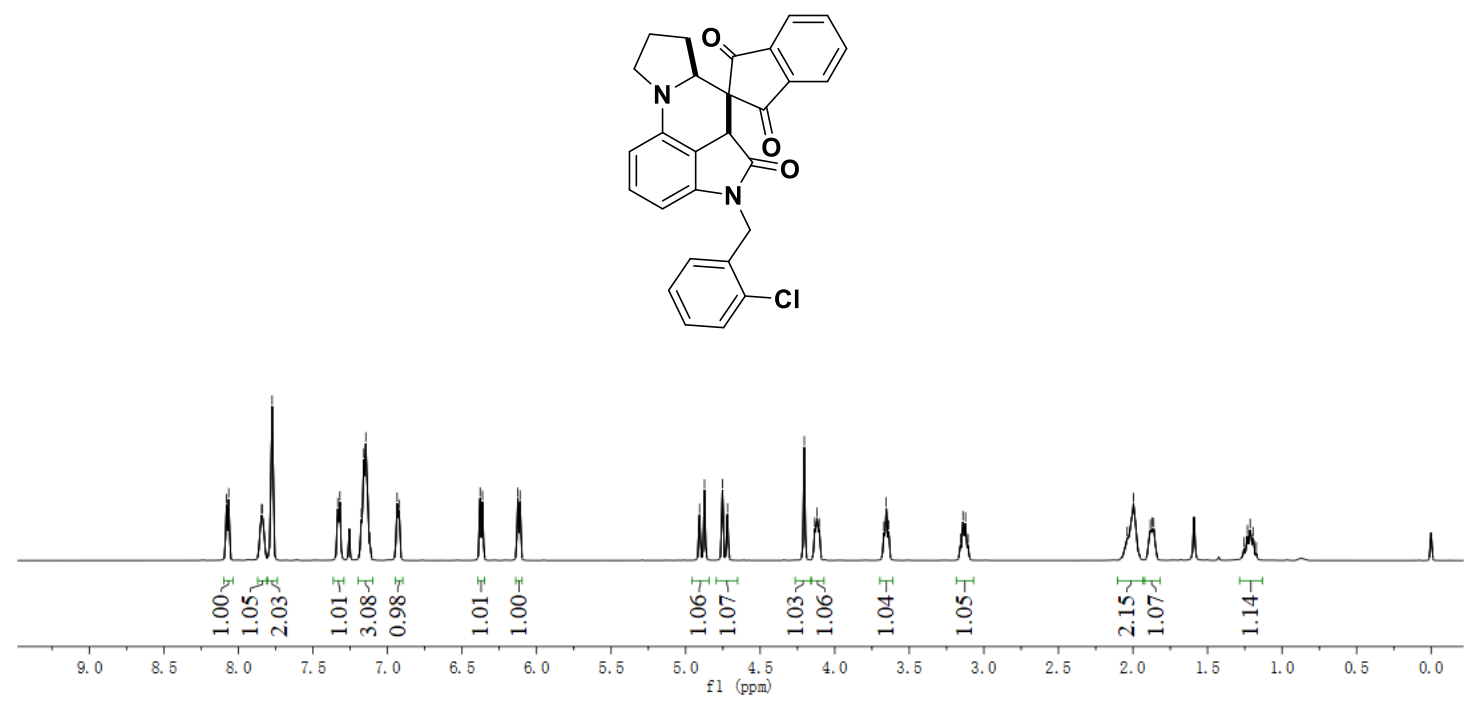

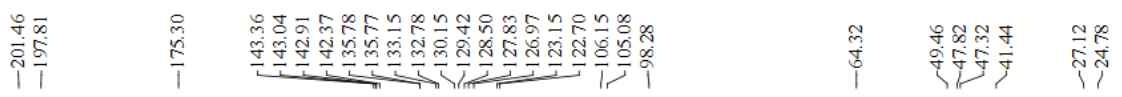
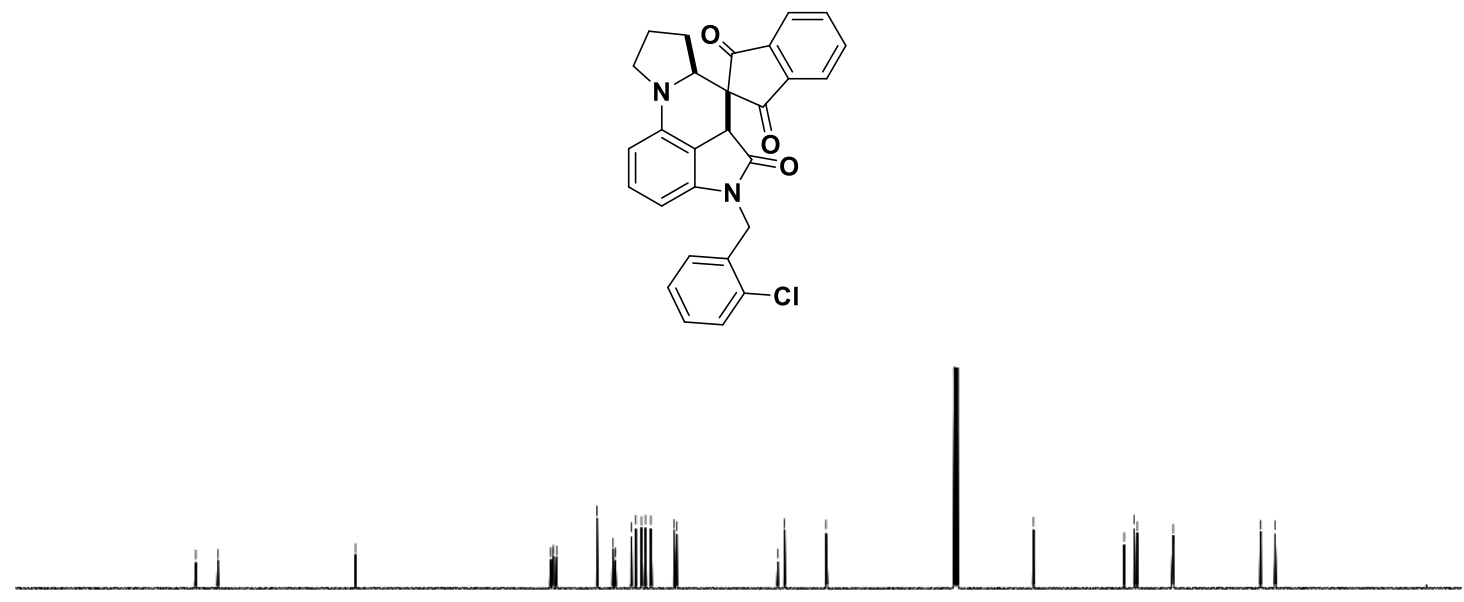

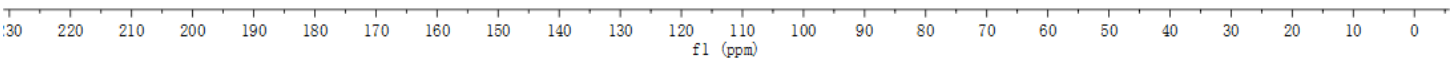


4-(4-bromobenzyl)-4,5a,6a,7,8,9-hexahydro-5H-spiro[dipyrrolo[1,2-a:4',3',2'-de]quinoline-6,2'-in dene]-1',3',5-trione (3c)

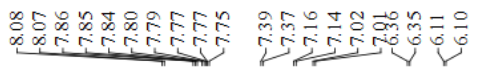
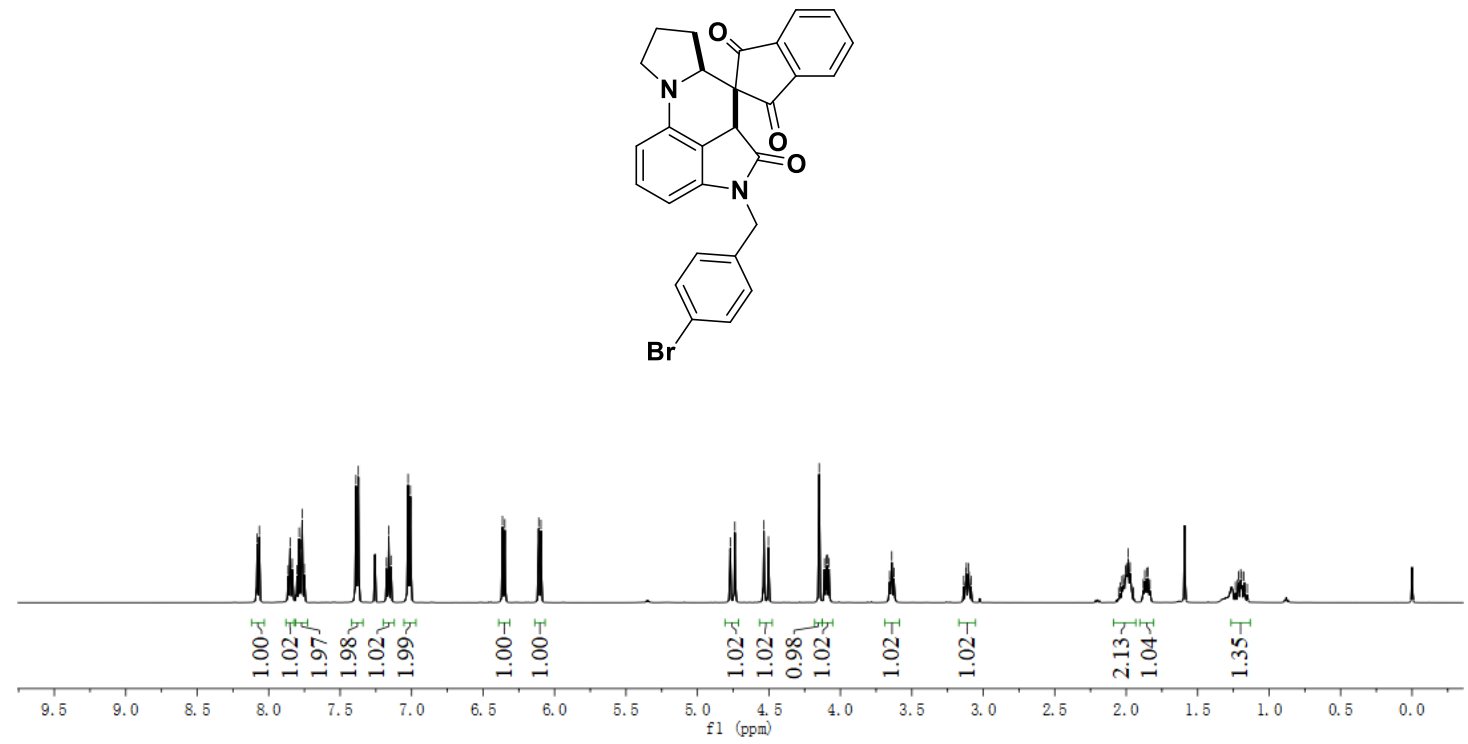

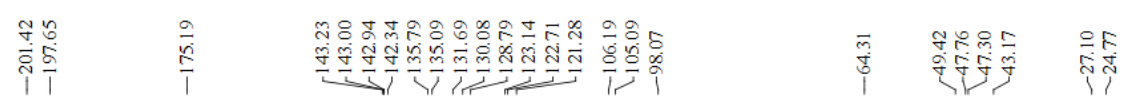

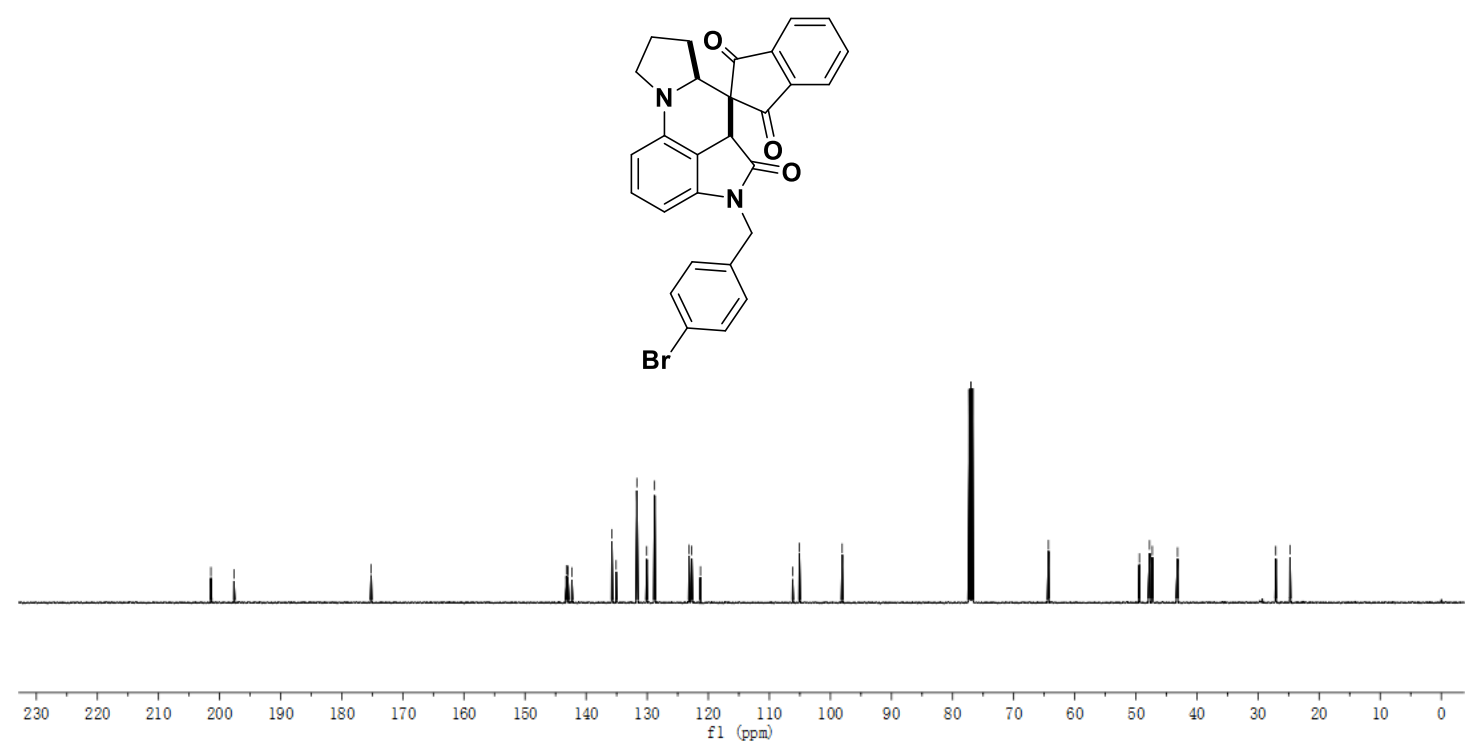


4-(4-methylbenzyl)-4,5a,6a,7,8,9-hexahydro-5H-spiro[dipyrrolo[1,2-a:4',3',2'-de]quinoline-6,2'-in dene]-1',3',5-trione (3d)
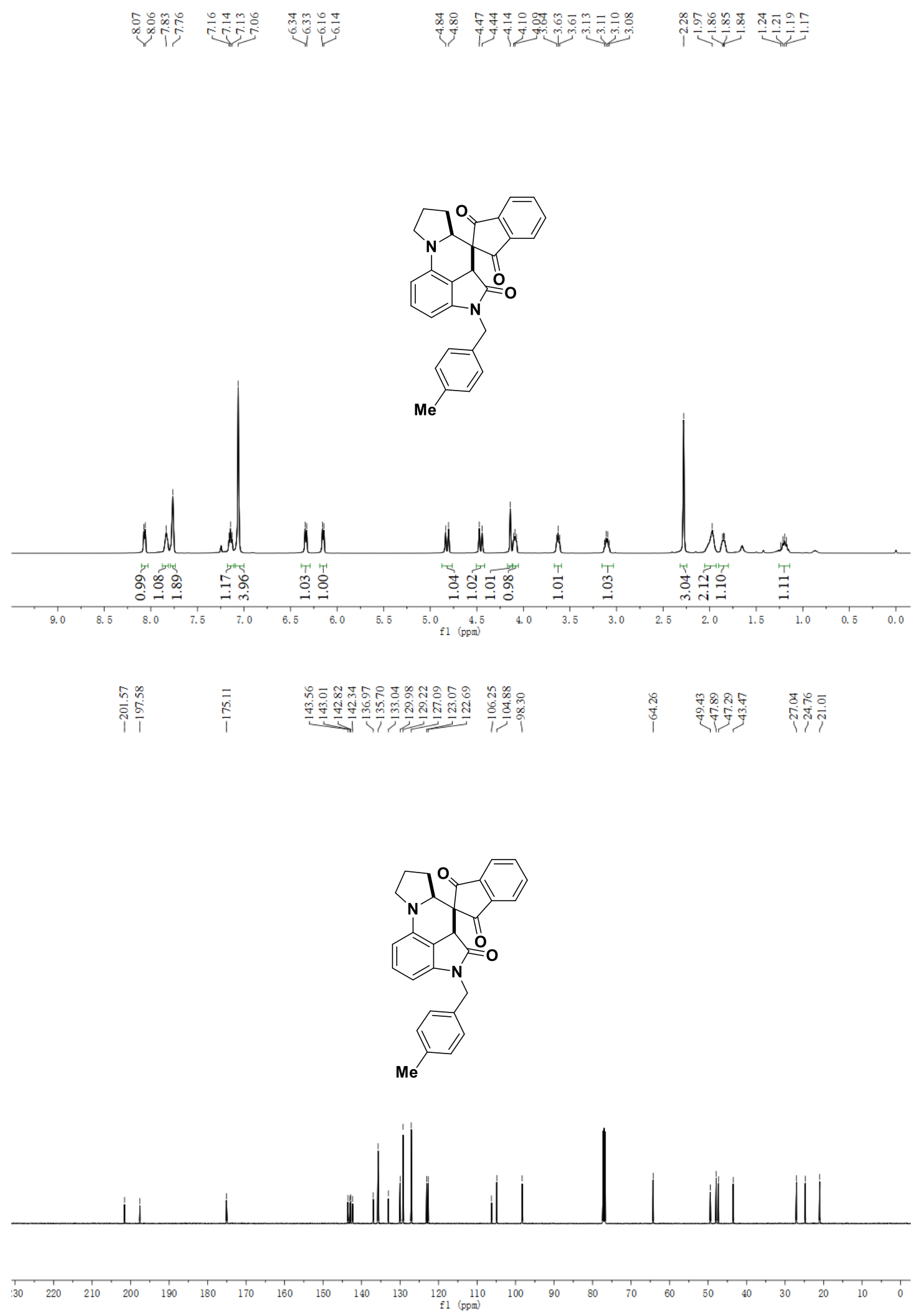
4-(naphthalen-2-ylmethyl)-4,5a,6a,7,8,9-hexahydro-5H-spiro[dipyrrolo[1,2-a:4',3',2'-de]quinoline -6,2'-indene]-1',3',5-trione (3e)

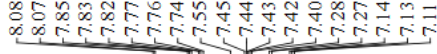

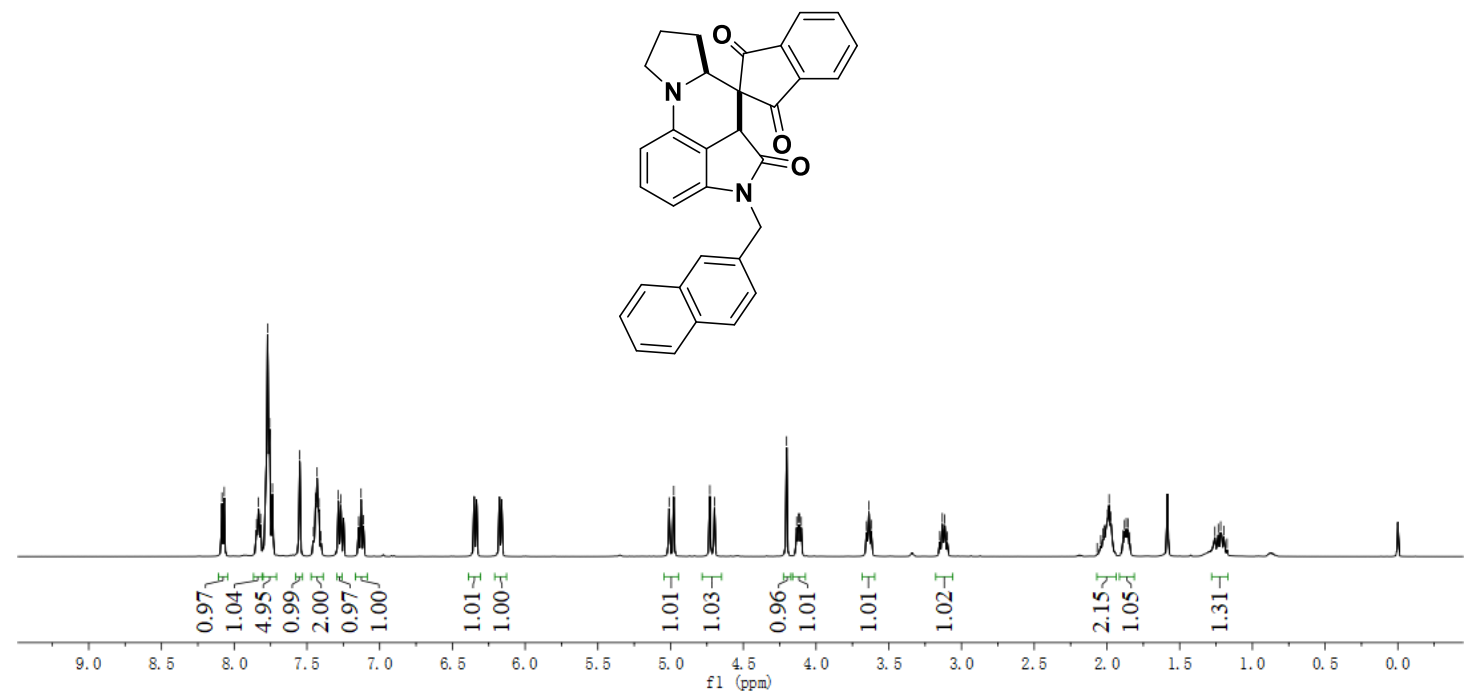

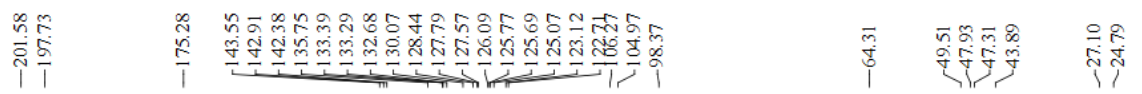

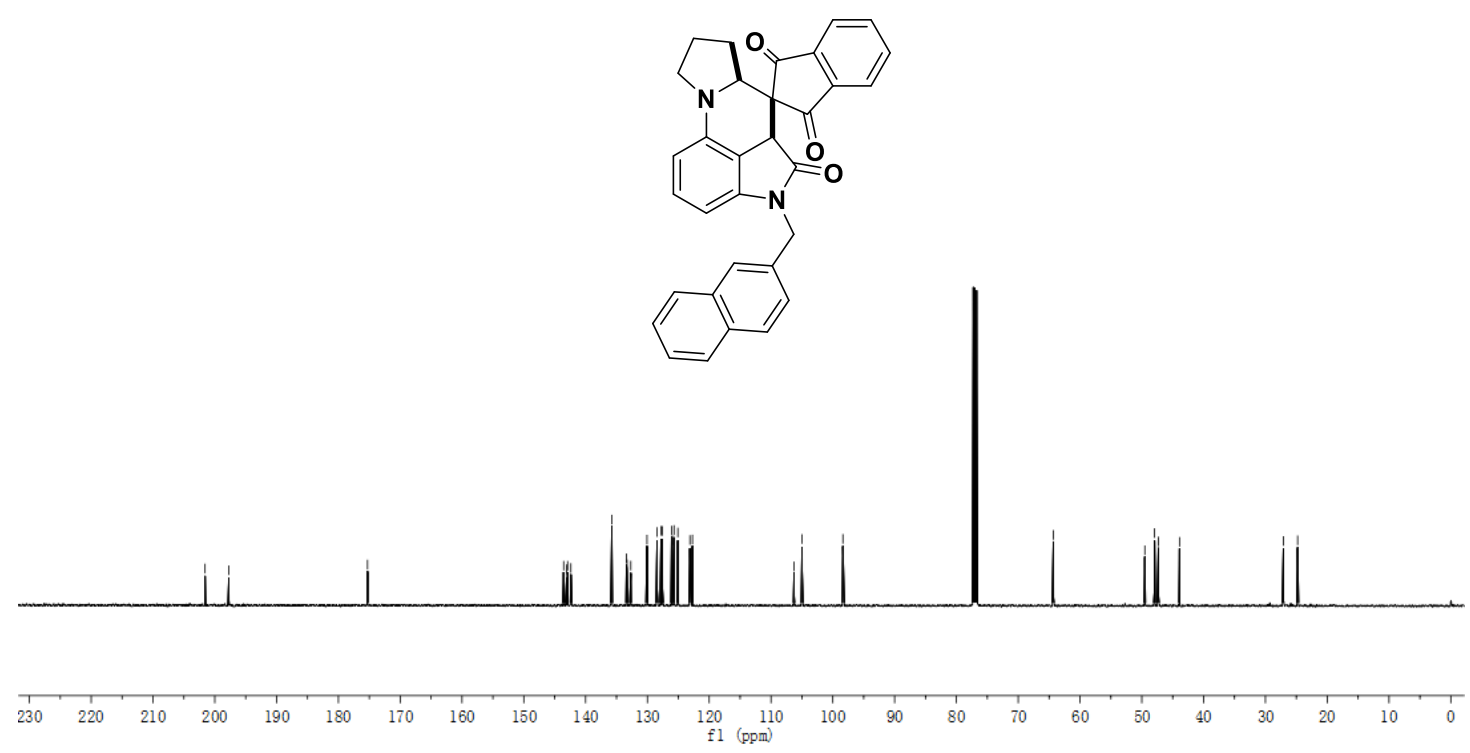


4-allyl-4,5a,6a,7,8,9-hexahydro-5H-spiro[dipyrrolo[1,2-a:4',3',2'-de]quinoline-6,2'-indene]-1',3',5trione (3f)

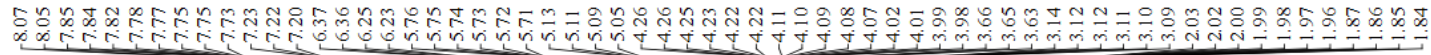
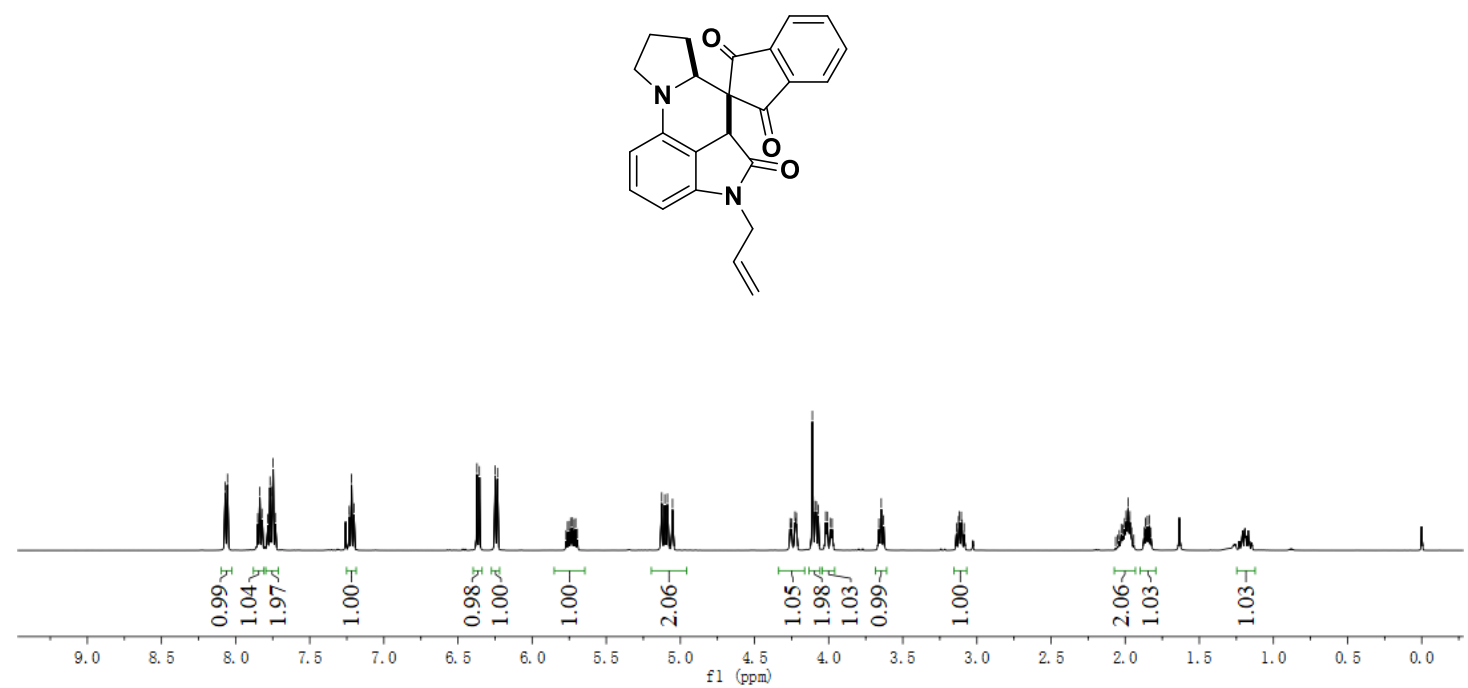

\begin{tabular}{|c|c|c|c|}
\hline 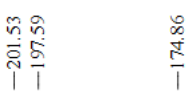 & 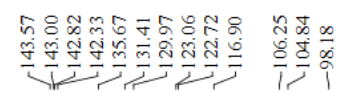 & $\begin{array}{c}0 \\
\substack{0 \\
\vdots \\
1}\end{array}$ & 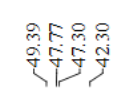 \\
\hline
\end{tabular}
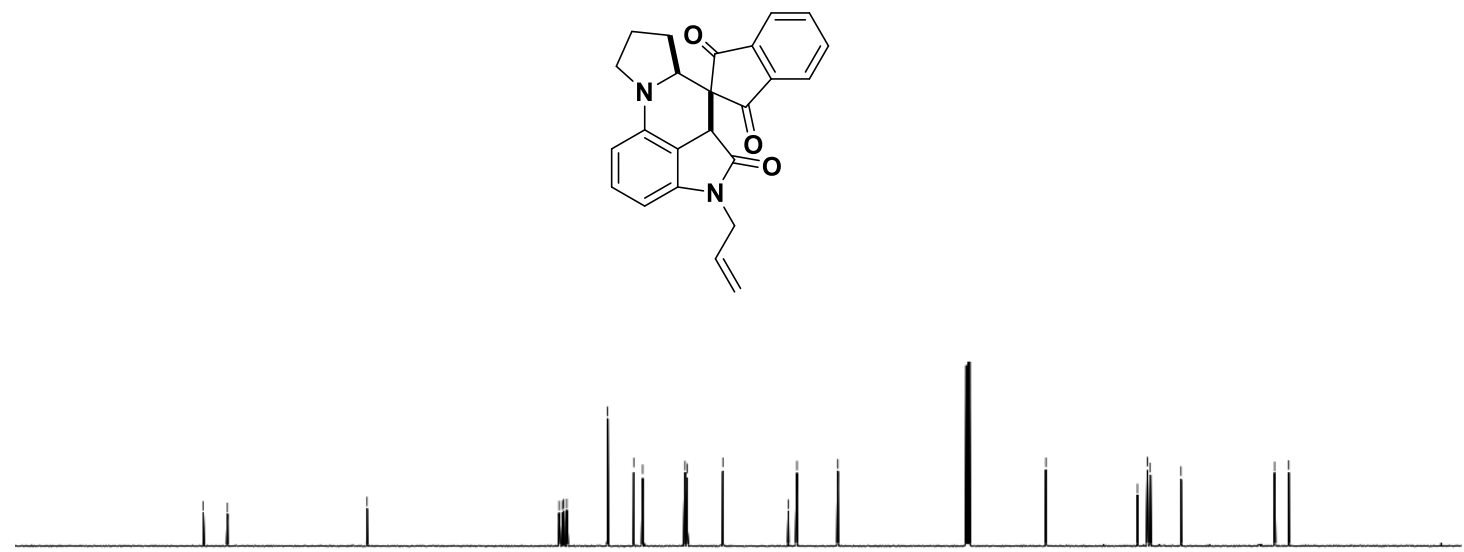

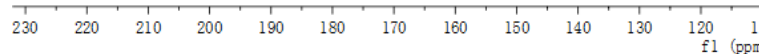


4-phenyl-4,5a,6a,7,8,9-hexahydro-5H-spiro[dipyrrolo[1,2-a:4',3',2'-de]quinoline-6,2'-indene]-1',3' ,5-trione $(3 g)$

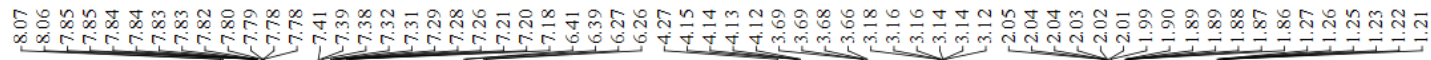
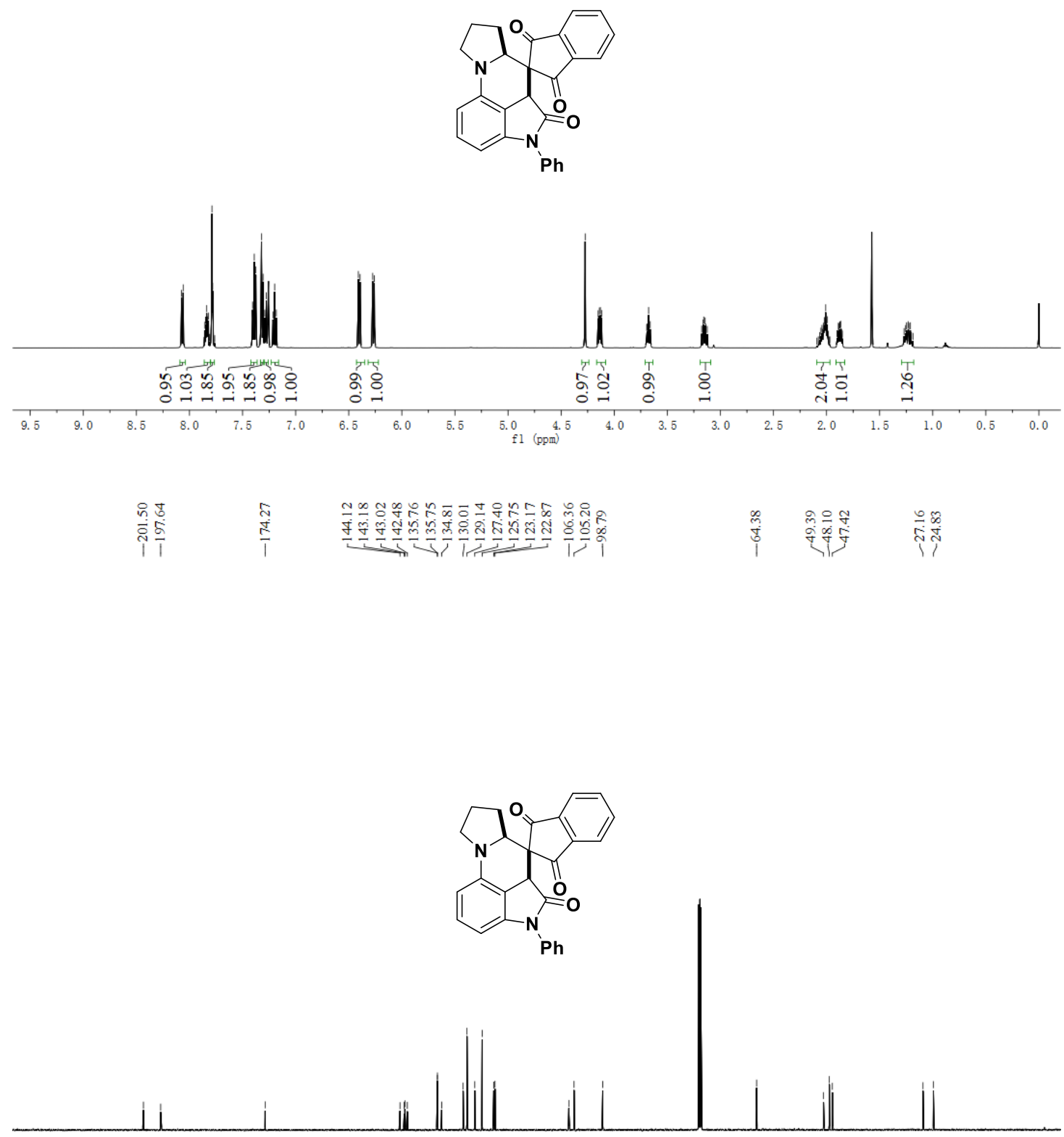

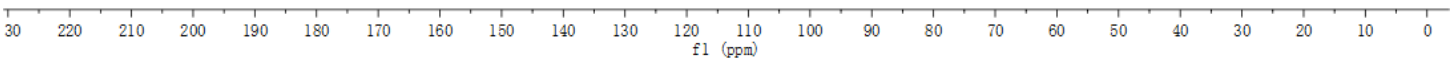


4-methyl-4,5a,6a,7,8,9-hexahydro-5H-spiro[dipyrrolo[1,2-a:4',3',2'-de]quinoline-6,2'-indene]-1',3' ,5-trione (3h)
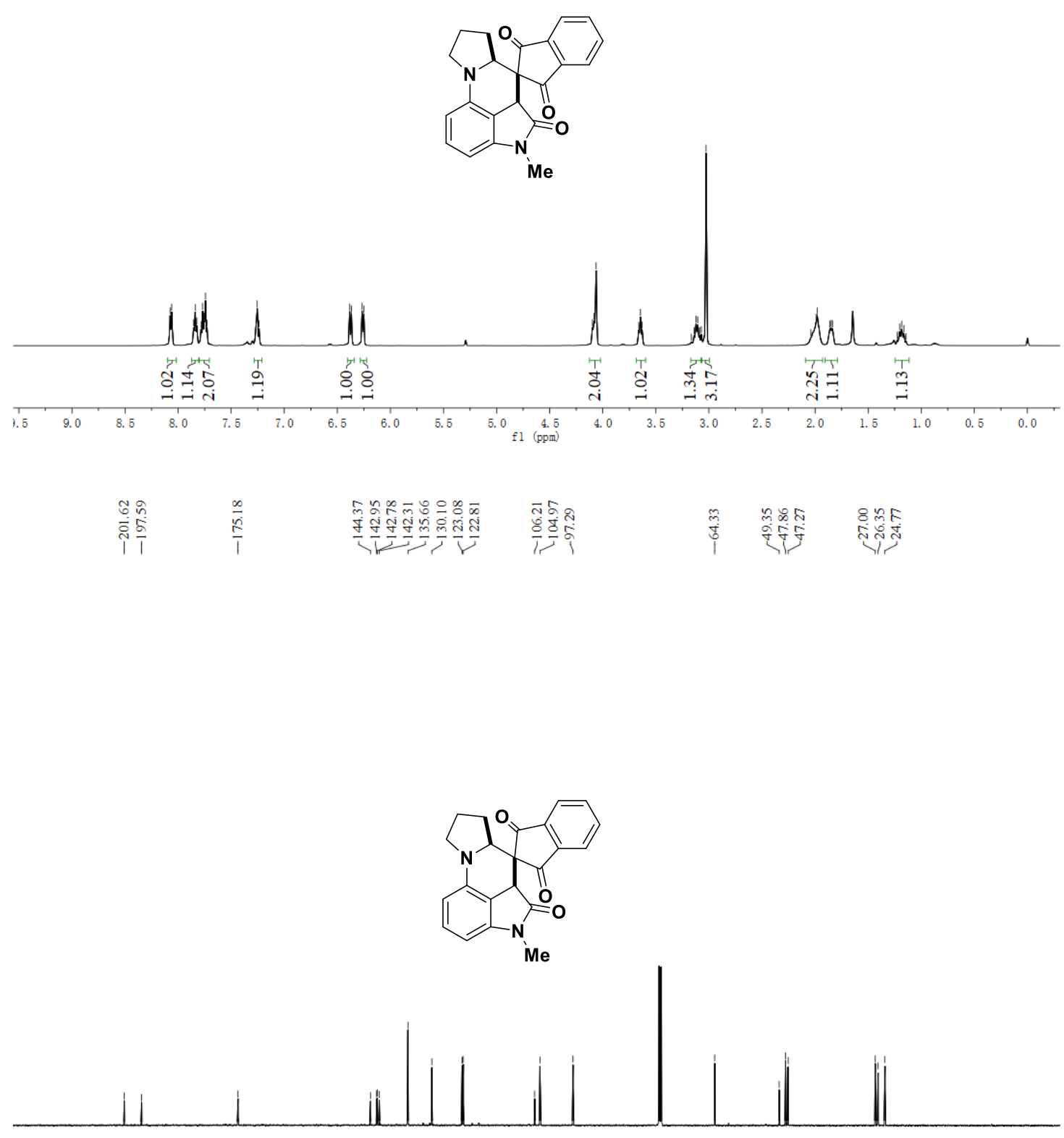

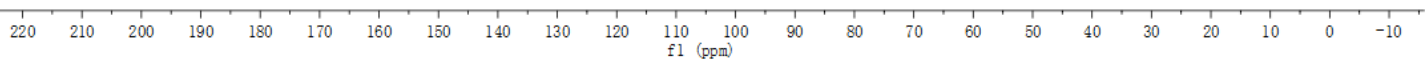


4-ethyl-4,5a,6a,7,8,9-hexahydro-5H-spiro[dipyrrolo[1,2-a:4',3',2'-de]quinoline-6,2'-indene]-1',3',5 -trione (3i)
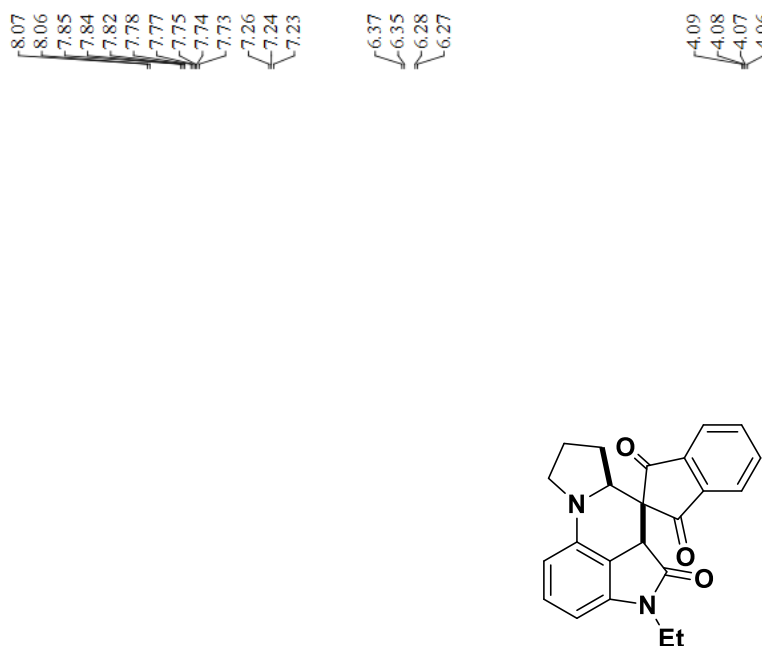

Et

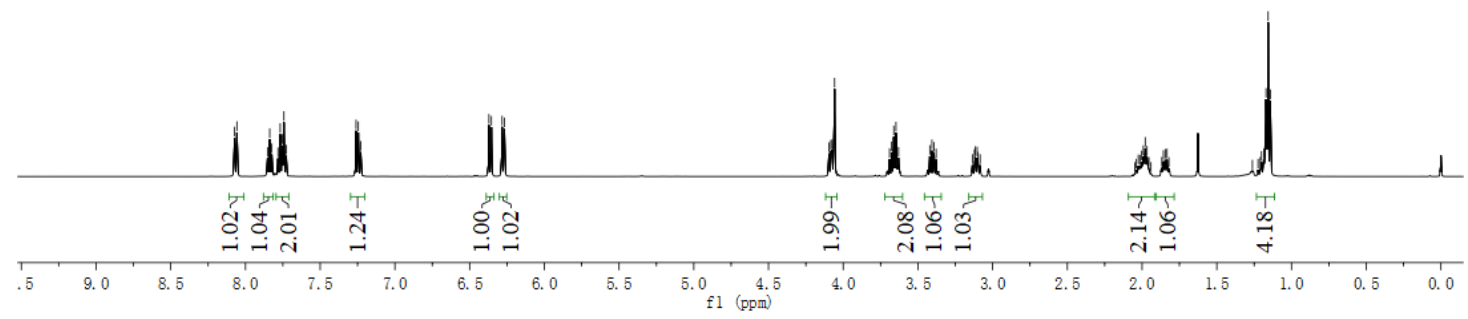

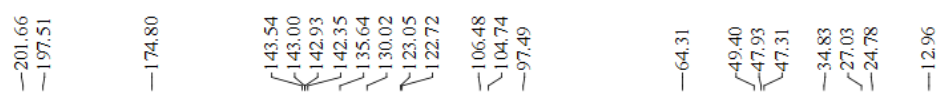
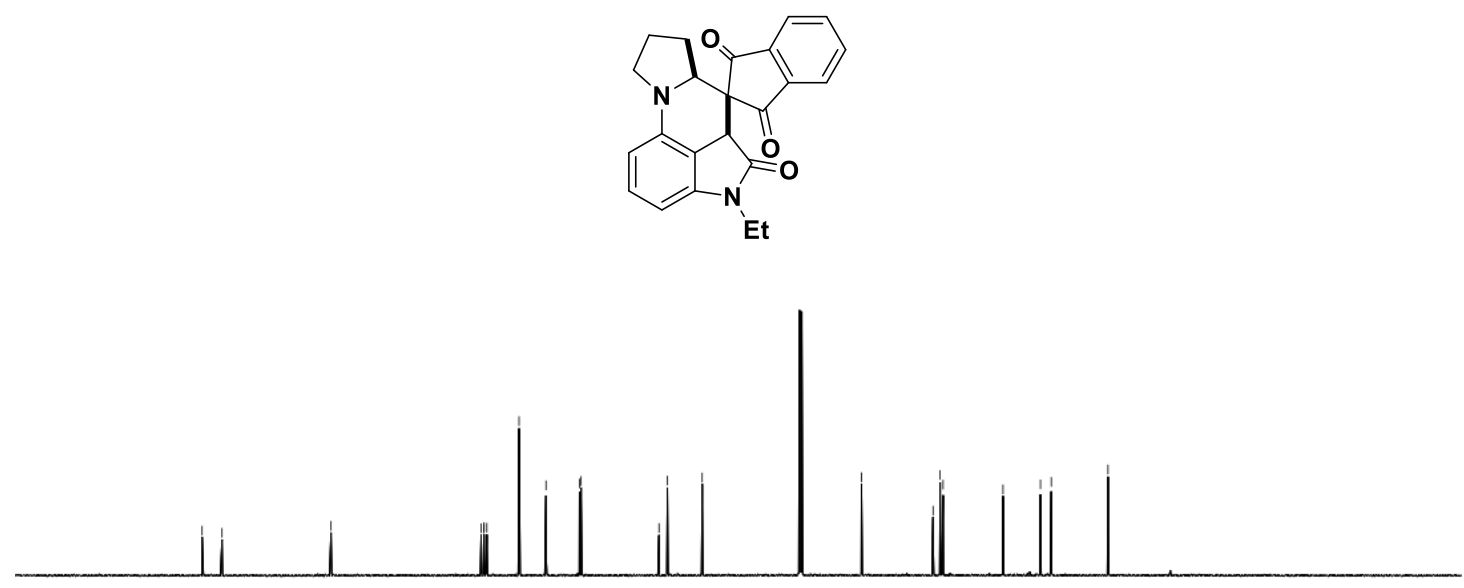

$\begin{array}{llllllllllllllllllllllllllllllllllllllll}40 & 230 & 220 & 210 & 200 & 190 & 180 & 170 & 160 & 150 & 140 & 130 & 120 & 110 & 100 & 90 & 80 & 70 & 60 & 50 & 40 & 30 & 20 & 10 & 0 & -10 & -20 & -30 & -40 & -50 & -6\end{array}$ 
4-(cyclopropylmethyl)-4,5a,6a,7,8,9-hexahydro-5H-spiro[dipyrrolo[1,2-a:4',3',2'-de]quinoline-6,2' -indene]-1',3',5-trione (3j)

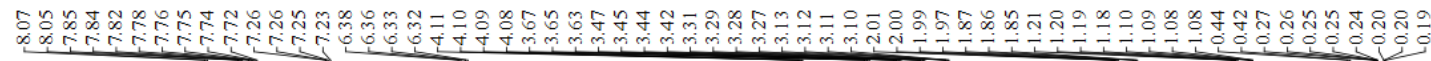
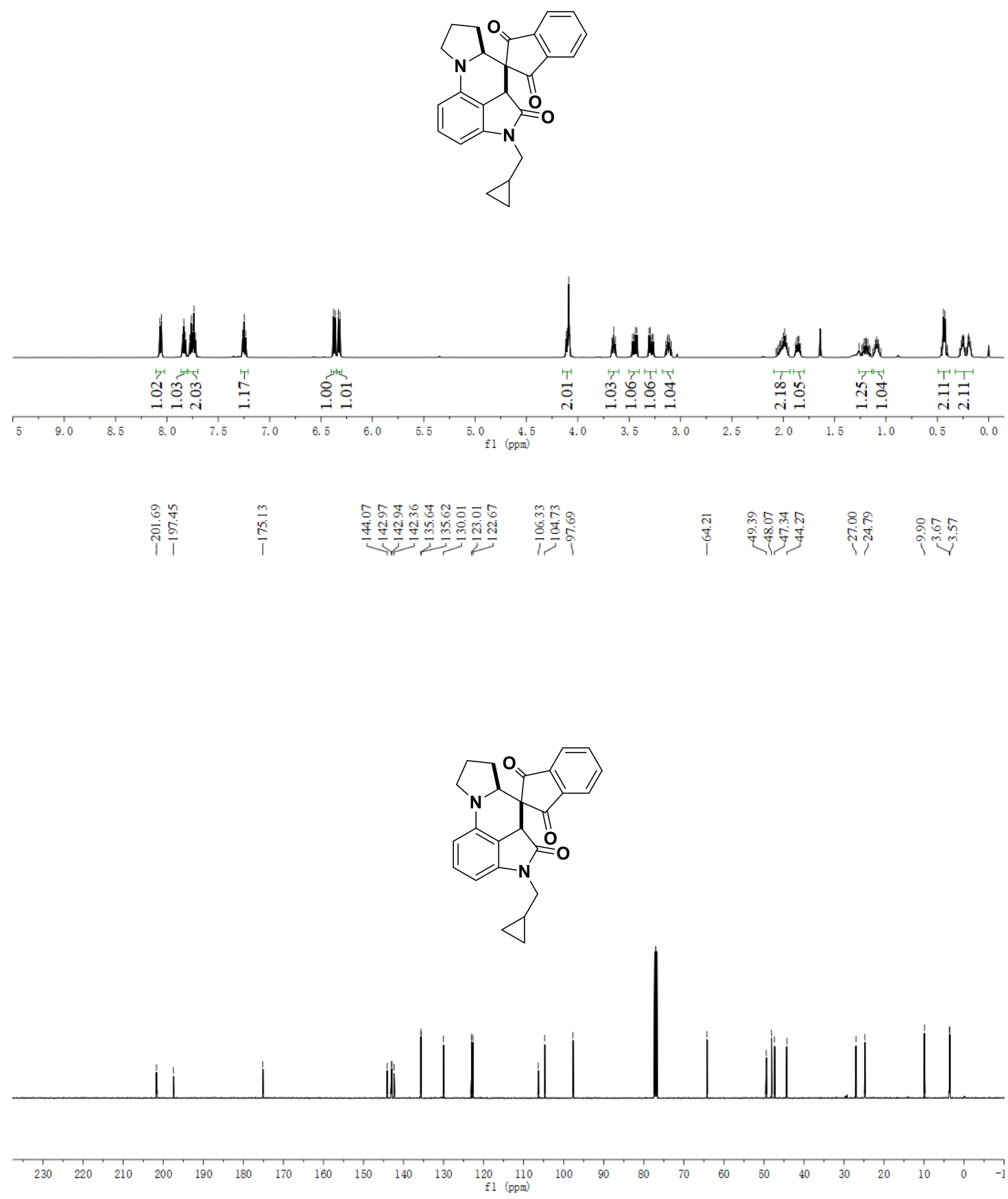

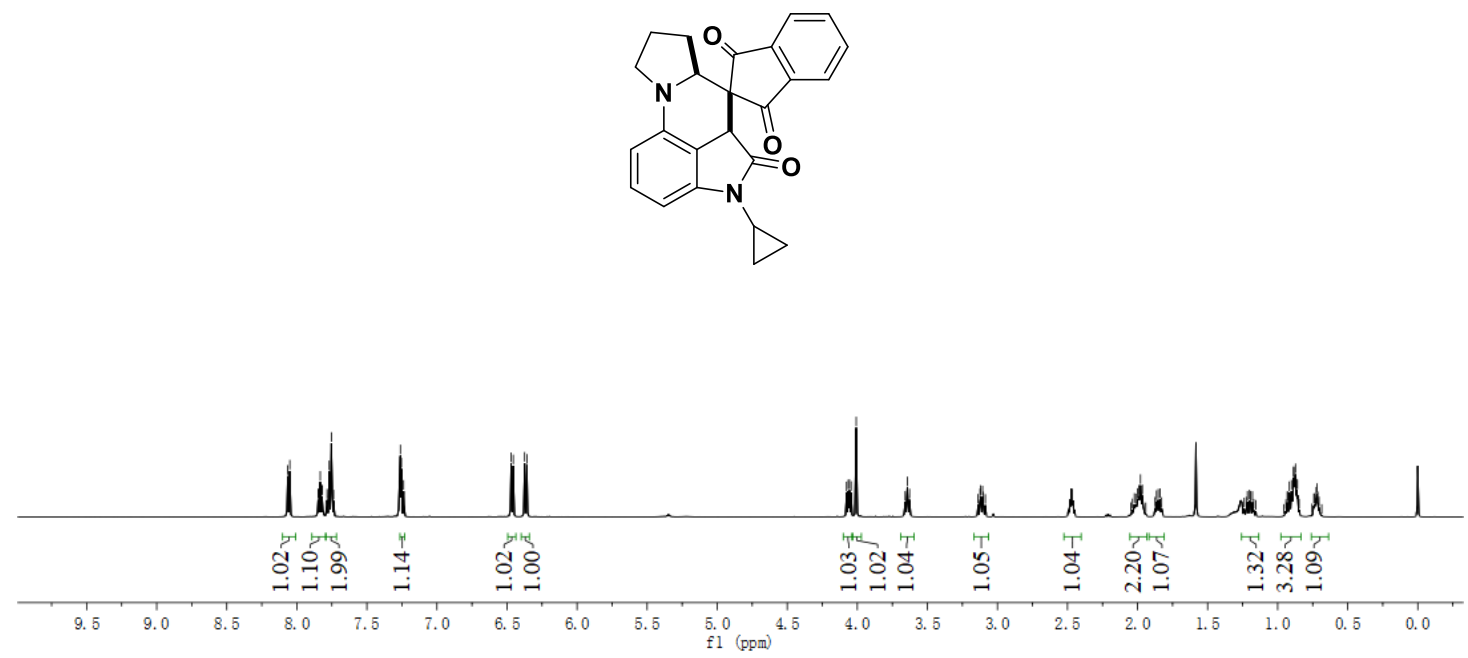

\begin{tabular}{|c|c|c|c|c|c|c|}
\hline & $\begin{array}{l}\overline{7} \\
0 \\
0 \\
1\end{array}$ & 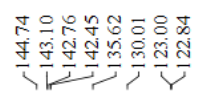 & 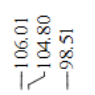 & $\frac{0}{0}$ & 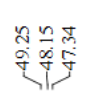 & 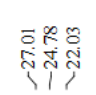 \\
\hline
\end{tabular}
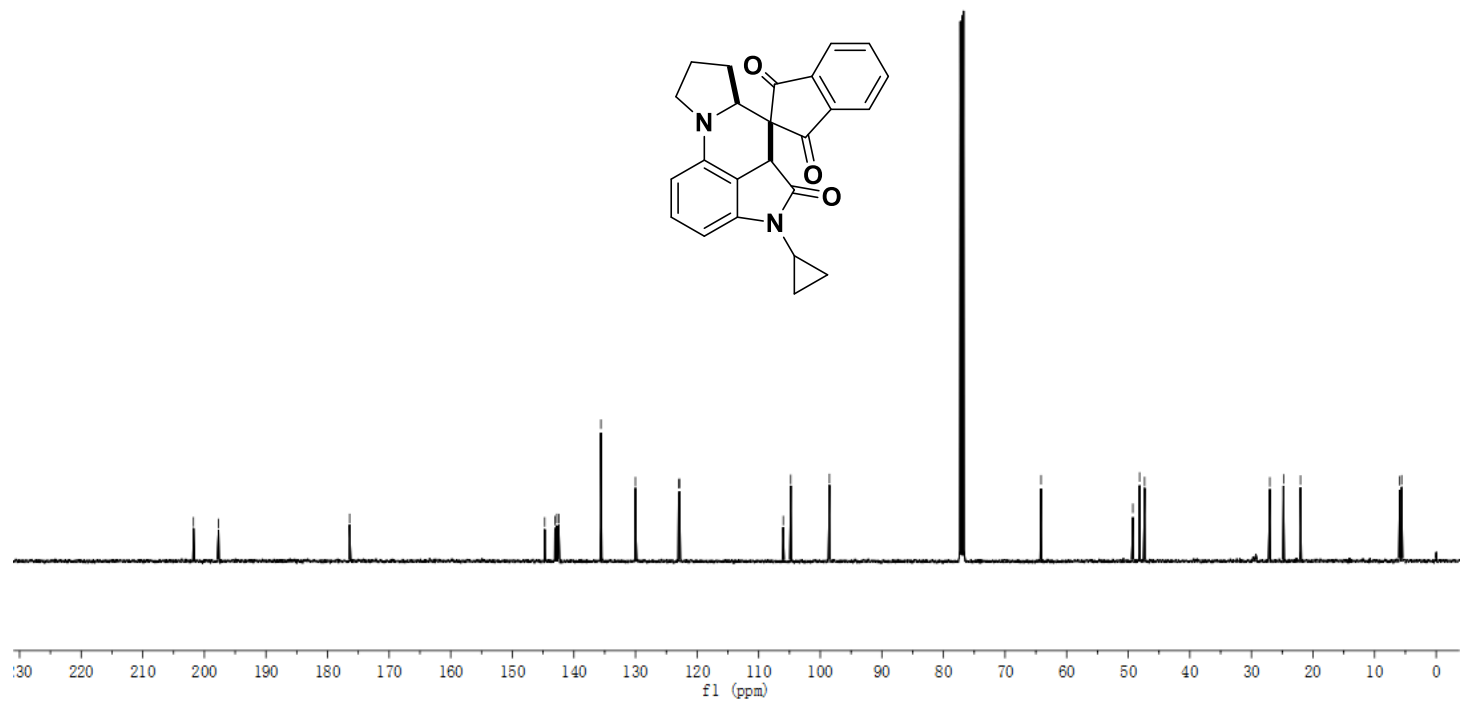
ethyl1',3',5-trioxo-1',3',5,5a,6a,7,8,9-octahydro-4H-spiro[dipyrrolo[1,2-a:4',3',2'-de]quinoline-6,2' -indene]-4-carboxylate (3I)

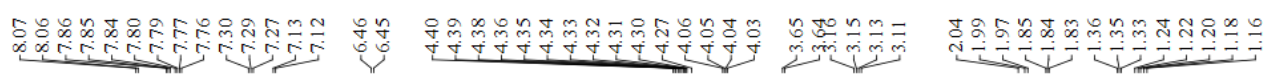
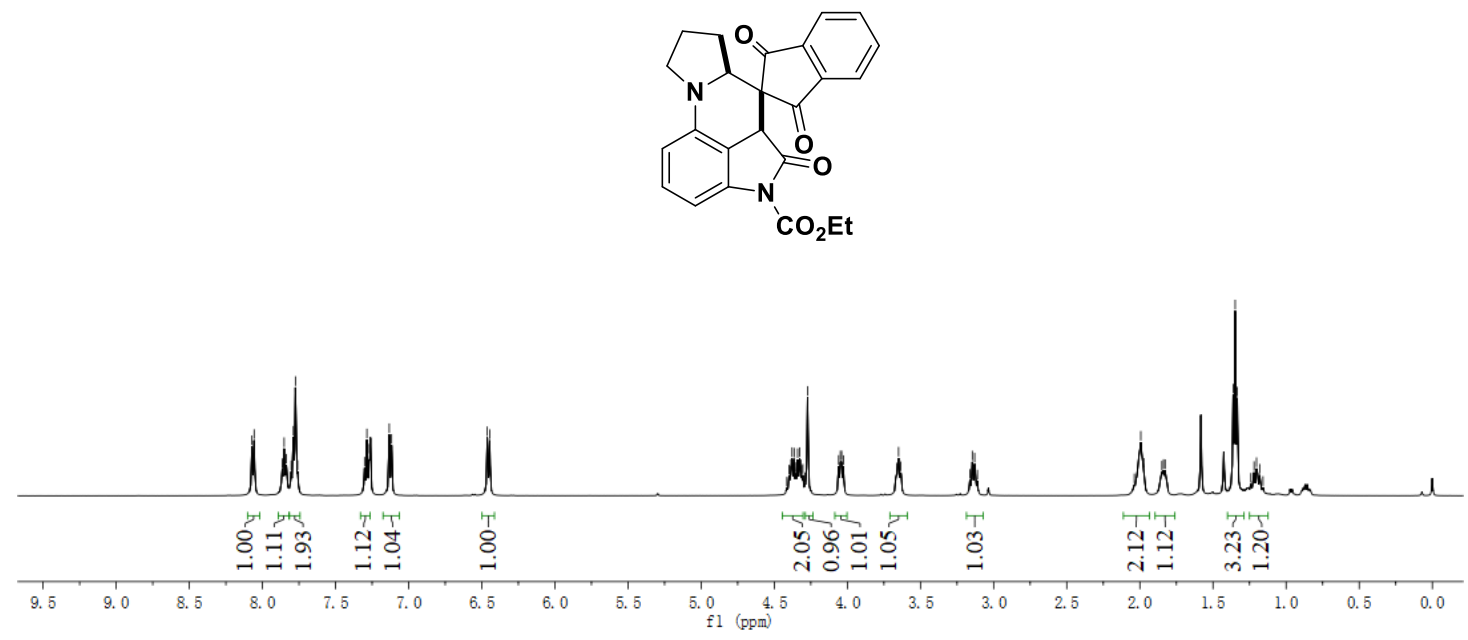

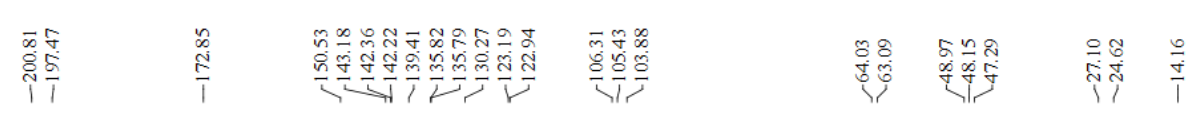
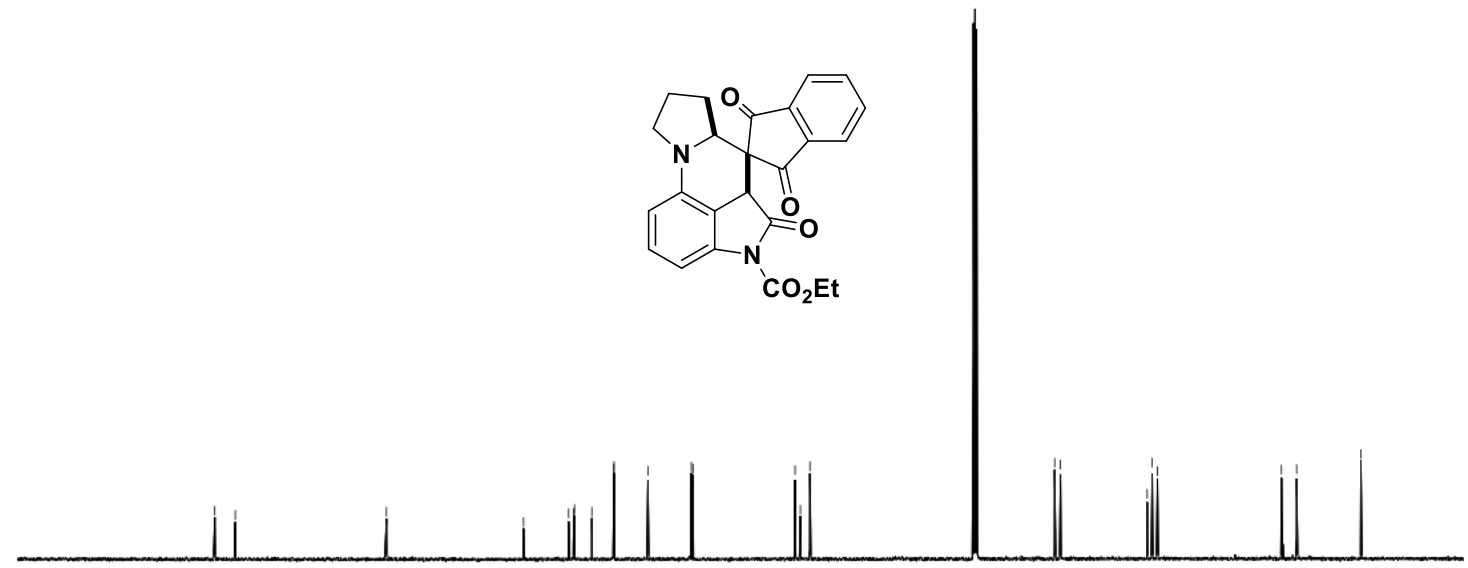

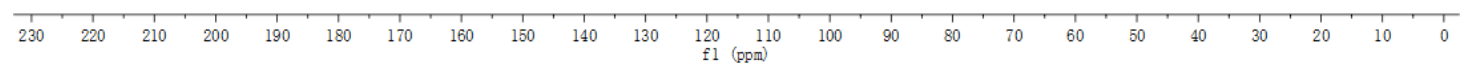


4,5a,6a,7,8,9-hexahydro-5H-spiro[dipyrrolo[1,2-a:4',3',2'-de]quinoline-6,2'-indene]-1',3',5-trione (3m)

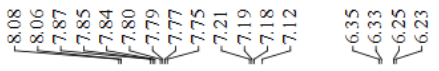
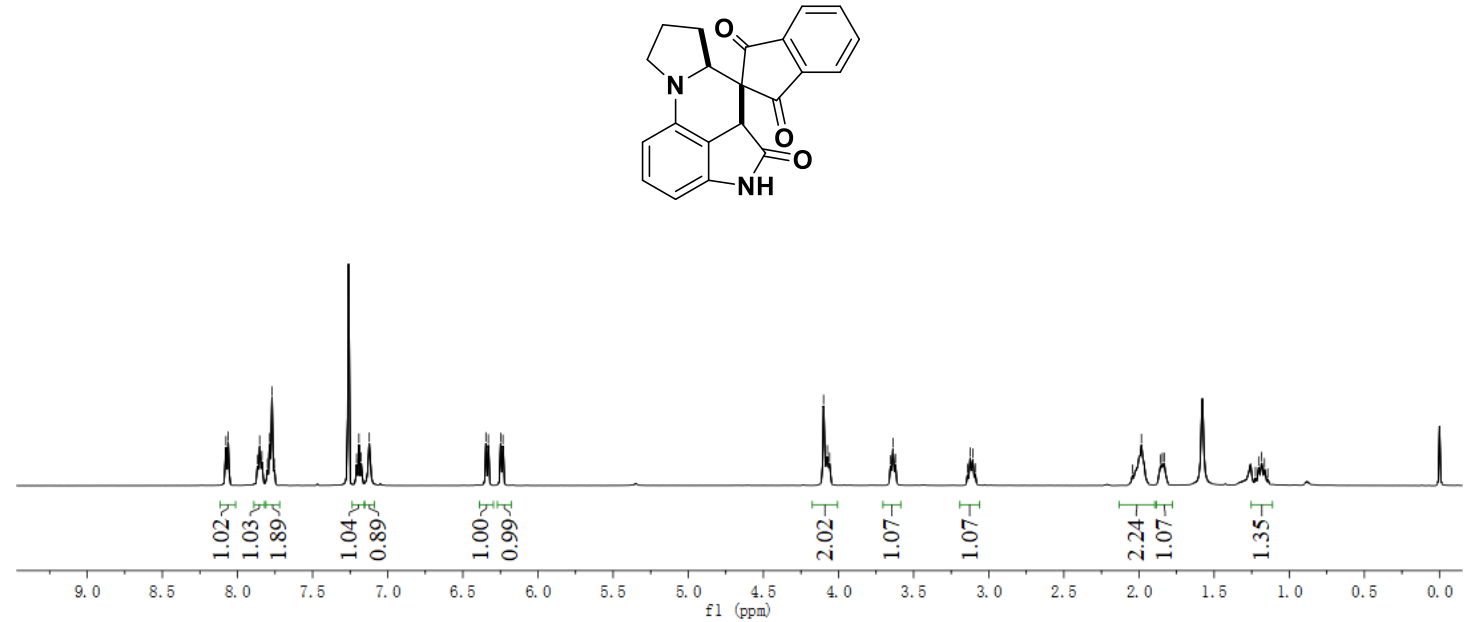

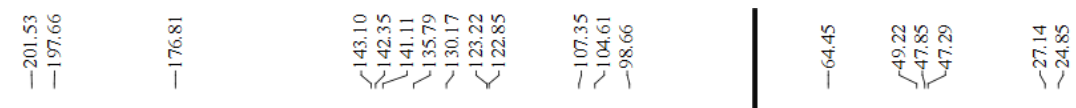

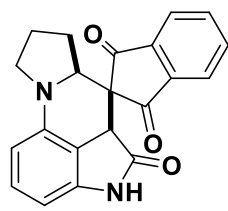

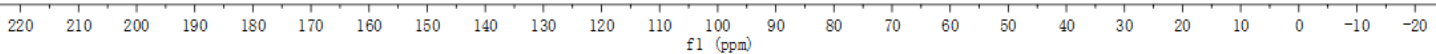


4-benzyl-1-methyl-4,5a,6a,7,8,9-hexahydro-5H-spiro[dipyrrolo[1,2-a:4',3',2'-de]quinoline-6,2'-ind ene]-1',3',5-trione (3n)

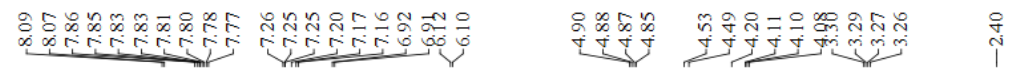
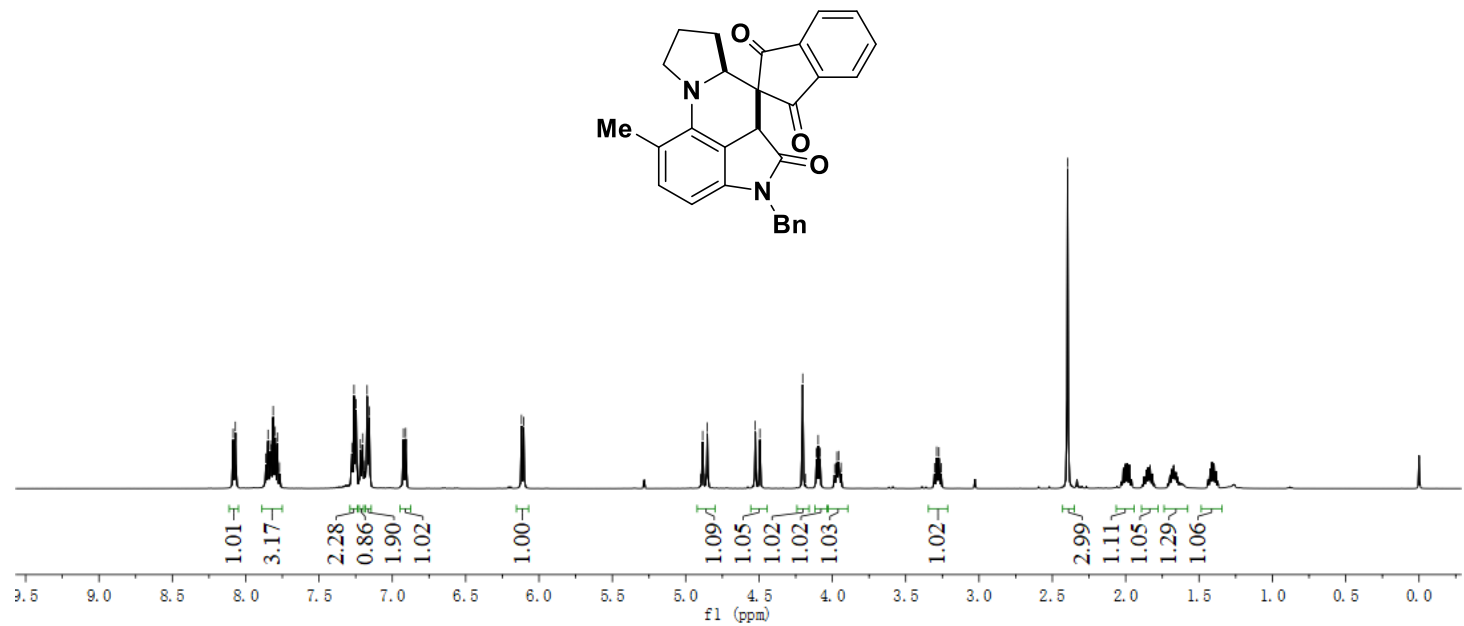

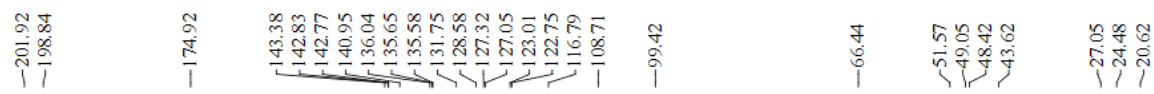
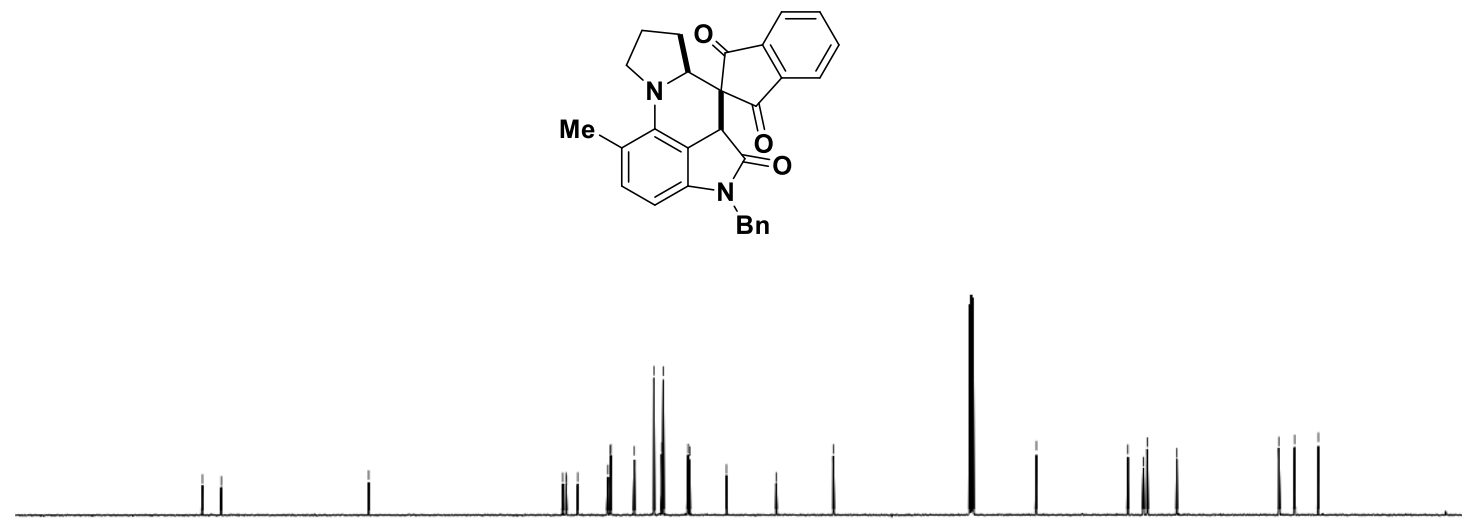

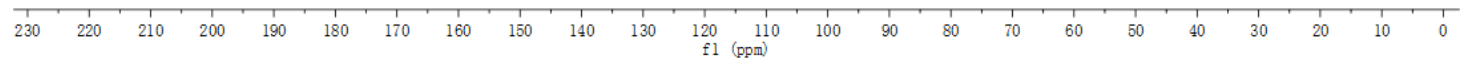


4-benzyl-3-chloro-4,5a,6a,7,8,9-hexahydro-5H-spiro[dipyrrolo[1,2-a:4',3',2'-de]quinoline-6,2'-ind ene]-1',3',5-trione (3o)

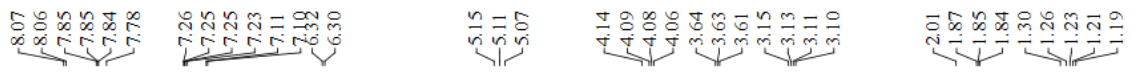
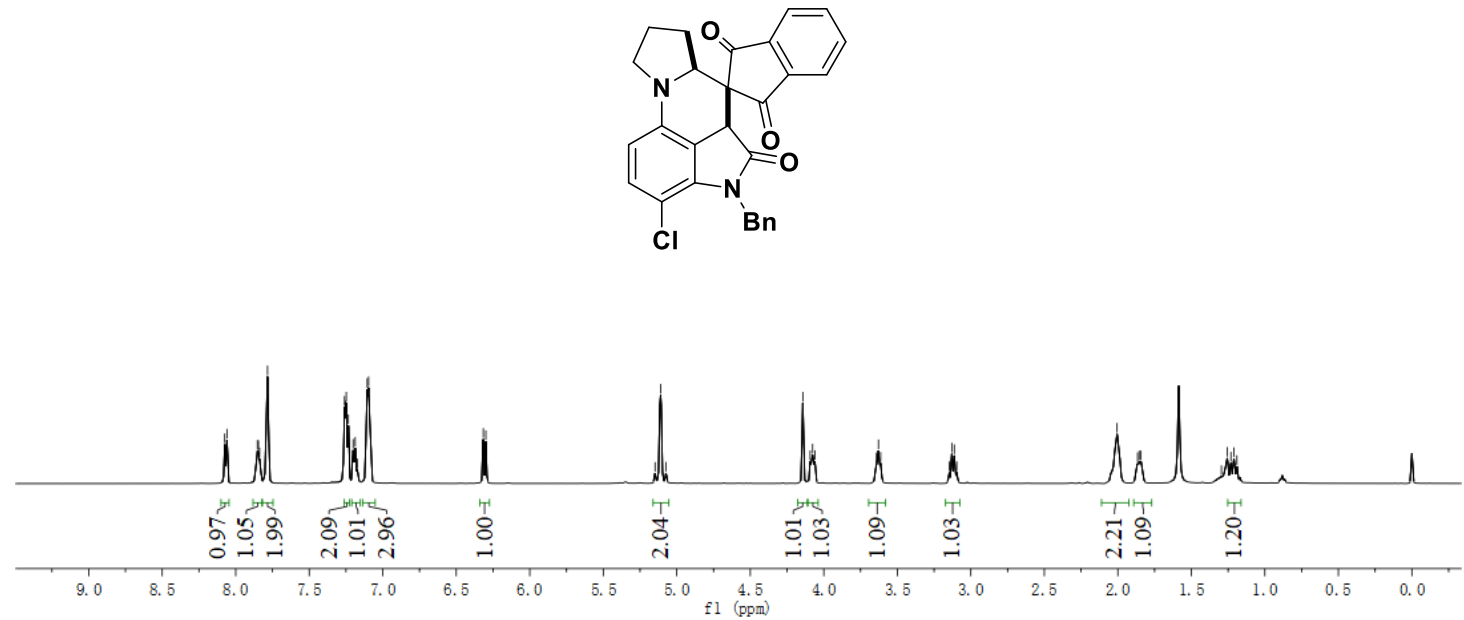

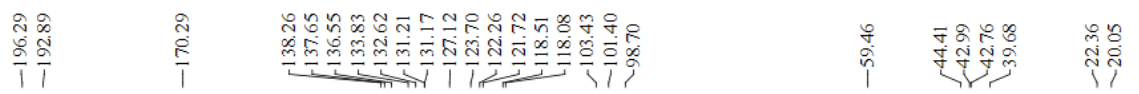
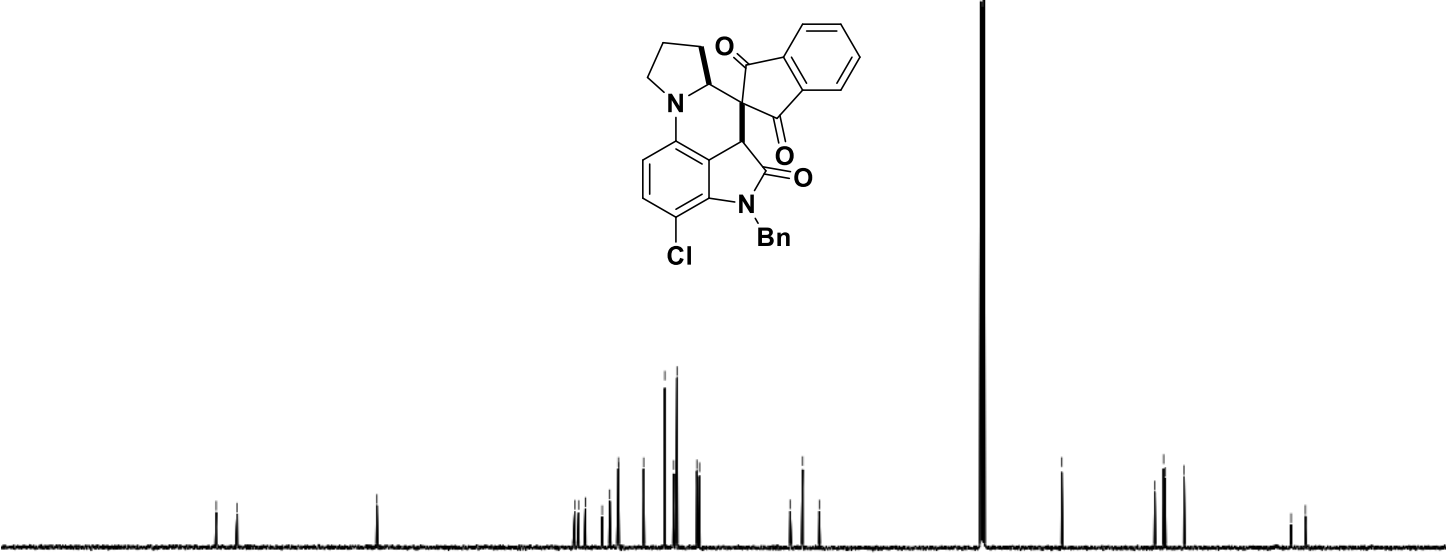

$\begin{array}{lllllllllllllllllllllllllll}130 & 220 & 210 & 200 & 190 & 180 & 170 & 160 & 150 & 140 & 130 & 120 & 110 & 100 & 90 & 80 & 70 & 60 & 50 & 40 & 30 & 20 & 10 & 0\end{array}$ 
4'-benzyl-4',5a',6a',6b',7',8',9',10',10a',11'-decahydro-5'H-spiro[indene-2,6'-isoindolo[2,1-a]pyrro lo[4,3,2-de]quinoline]-1,3,5'-trione (3p)

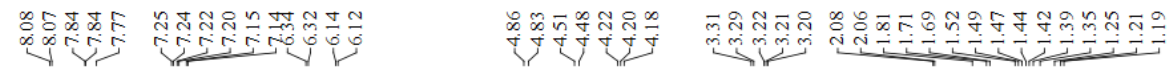
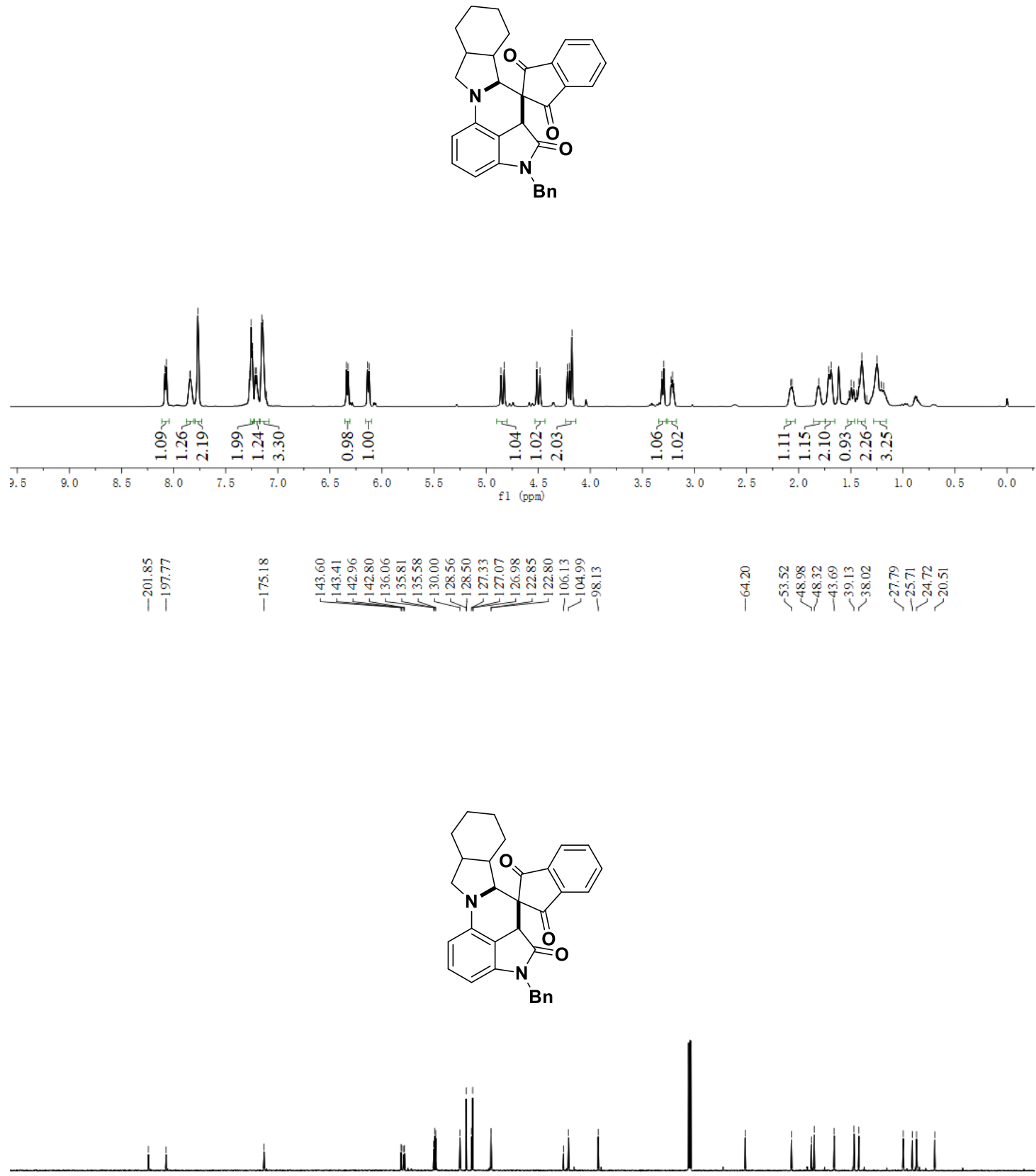

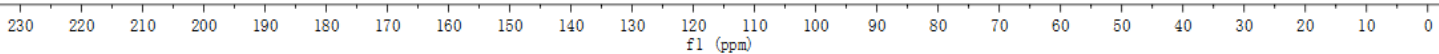


4'-benzyl-4',5a',6a',7',9',10'-hexahydro-5' $H$, 8' $^{\prime} H$-spiro[indene-2,6'-pyrido[1,2-a]pyrrolo[4,3,2-de]q uinoline]-1,3,5'-trione $(3 q)$

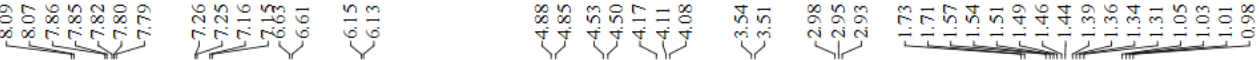
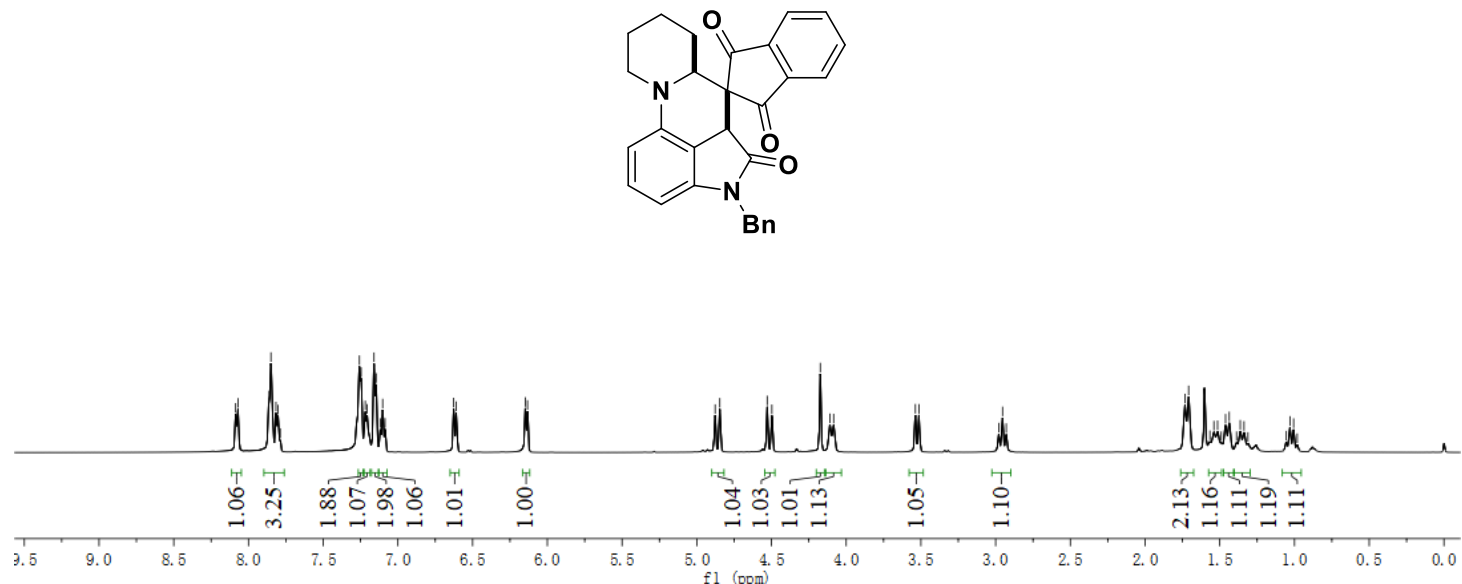

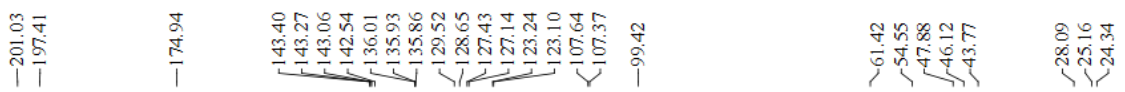
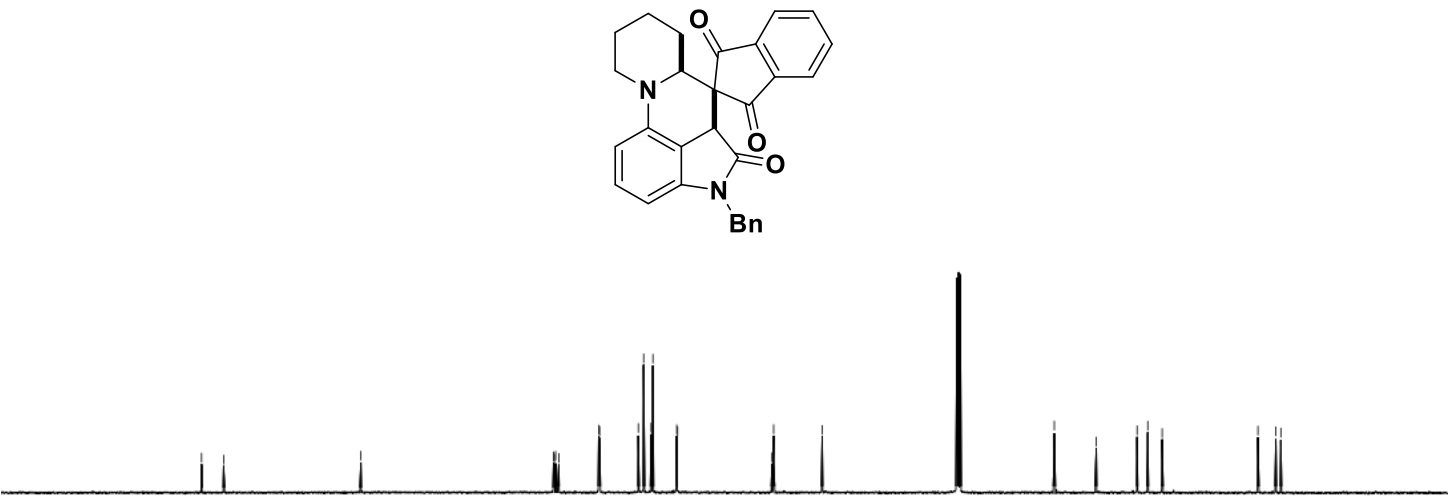

$\begin{array}{llllllllllllllllllllllllllllll}1 & 1 \\ 230 & 220 & 210 & 200 & 190 & 180 & 170 & 160 & 150 & 140 & 130 & \underset{f}{120} & 110 & 100 & 90 & 80 & 70 & 60 & 50 & 40 & 30 & 20 & 10 & 0\end{array}$ 
4'-benzyl-8'-methyl-4',5a',6a',7',9',10'-hexahydro-5' $H, 8$ ' $H$-spiro[indene-2,6'-pyrido[1,2-a]pyrrolo [4,3,2-de]quinoline]-1,3,5'-trione (3r)

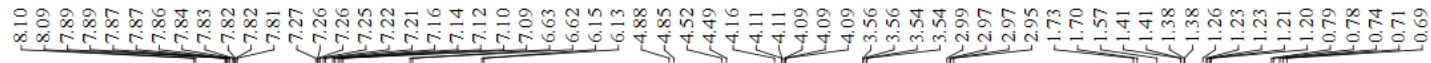<smiles>C[C@H]1CCN2C(=O)c3ccccc3C(=O)C3(C(=O)c4ccccc4)c4c(cccc42)C13</smiles>

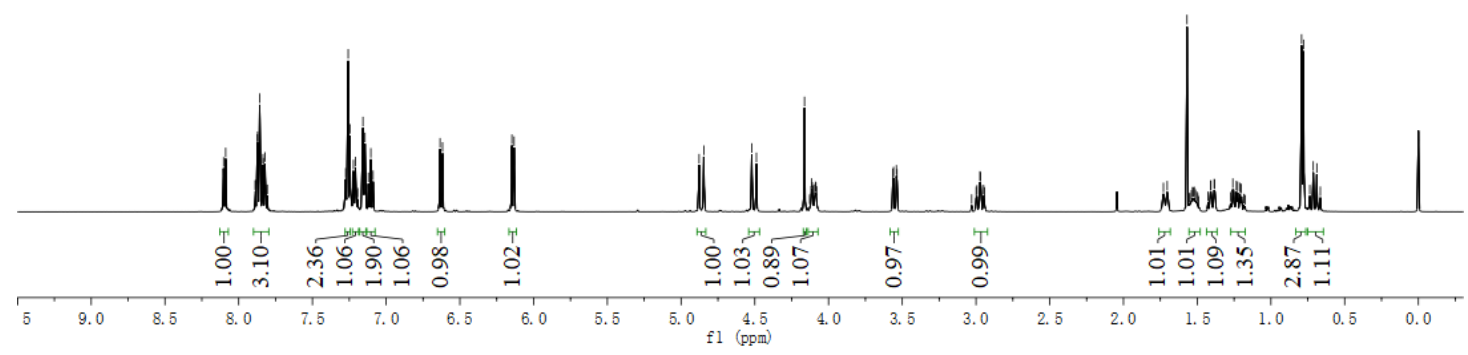

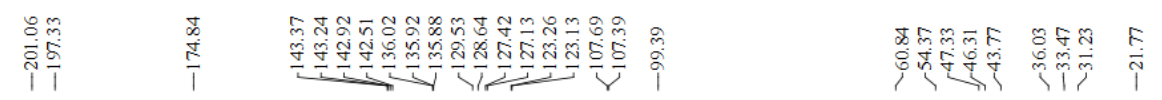
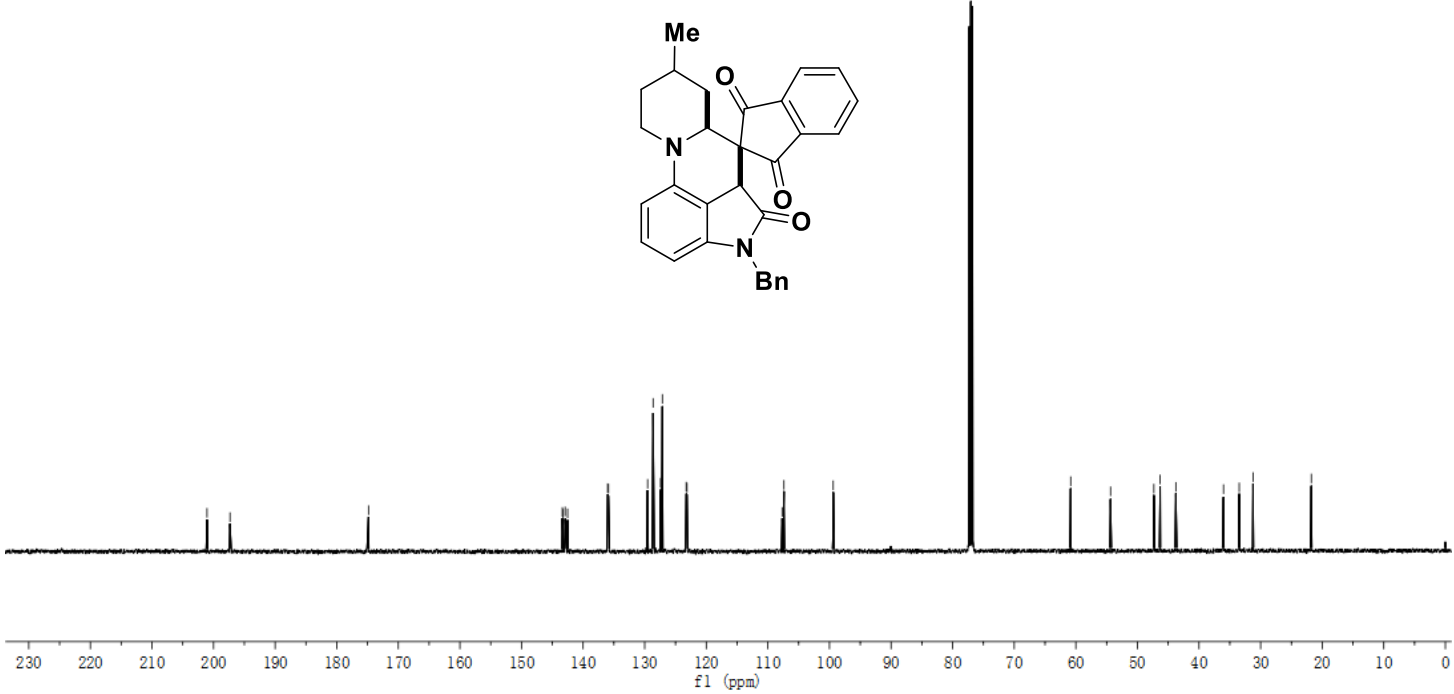
4'-benzyl-4',5a',6a',7',9',10'-hexahydro-5' $H$-spiro[indene-2,6'-[1,4]oxazino[4,3-a]pyrrolo[4,3,2-de] quinoline]-1,3,5'-trione $(3 \mathrm{~s})$

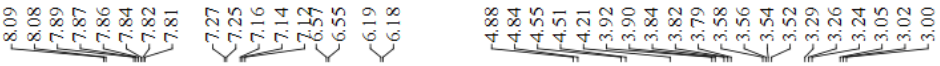
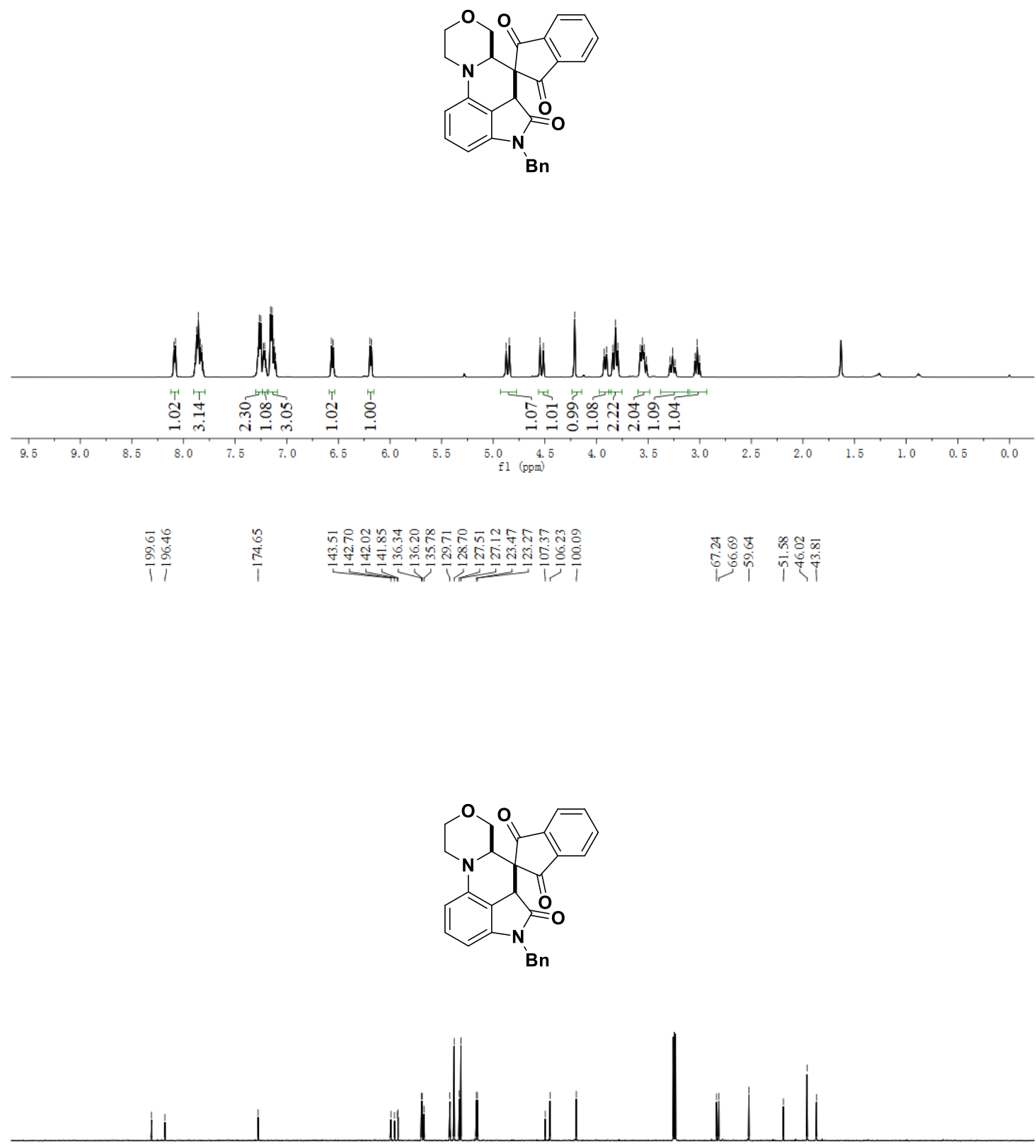

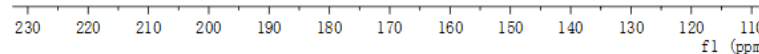


4'-benzyl-7',9'-dimethyl-4',5a',6a',7',9',10'-hexahydro-5' $H$-spiro[indene-2,6'-[1,4]oxazino[4,3-a]py rrolo[4,3,2-de]quinoline]-1,3,5'-trione (3t)

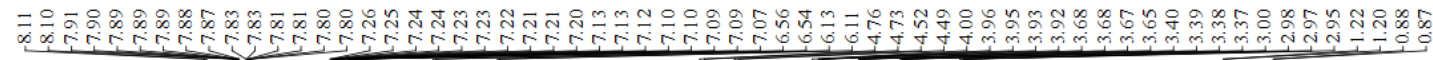
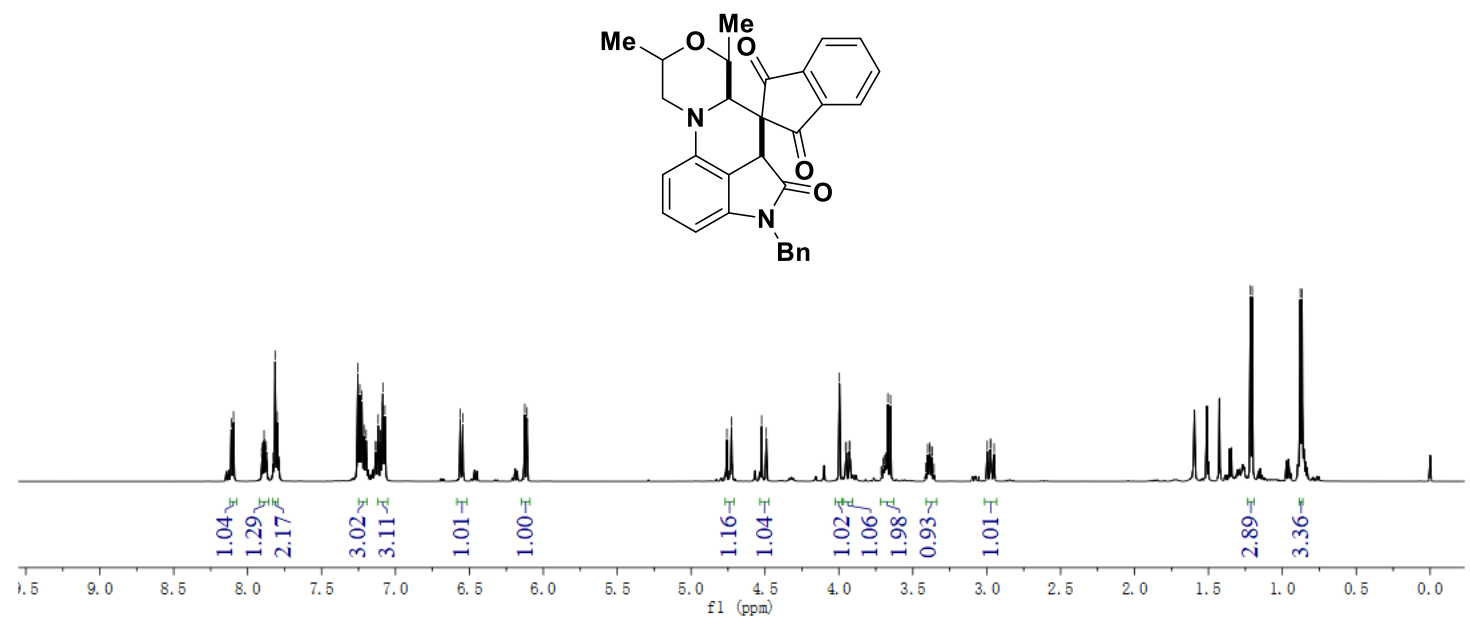

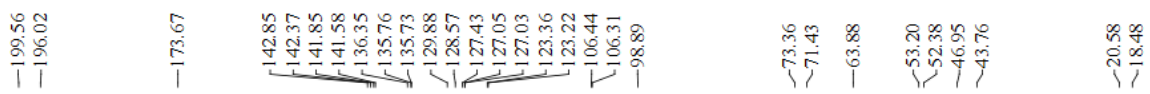
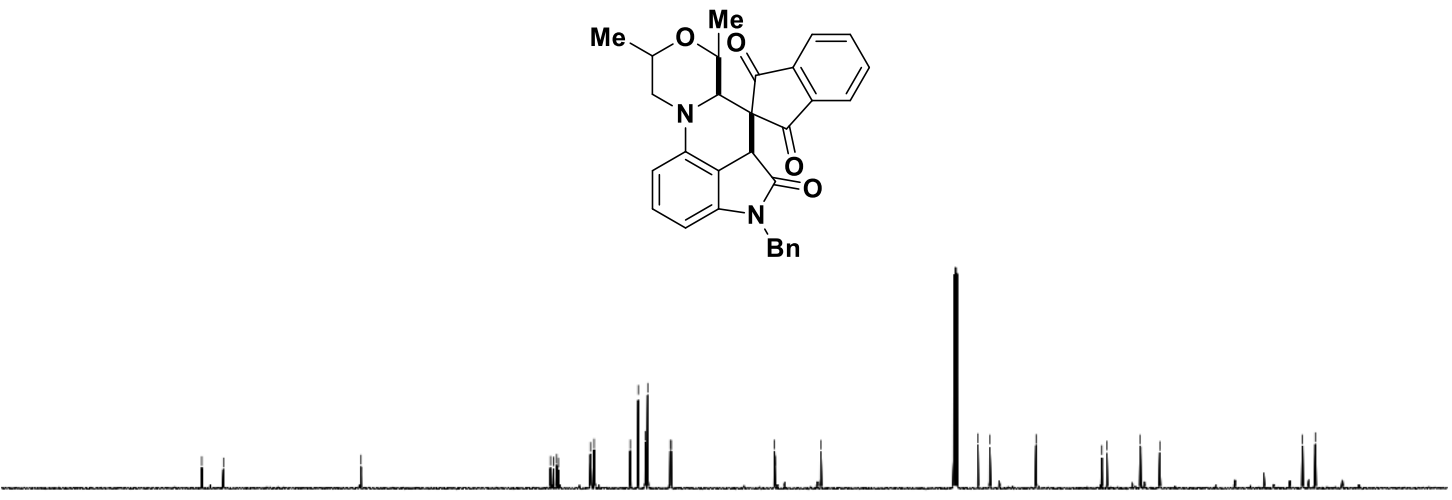

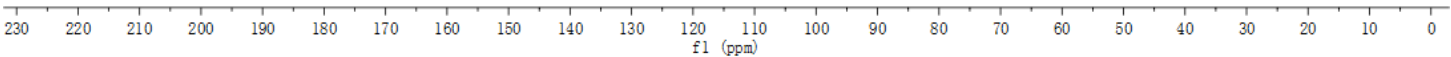


4'-benzyl-4',5a',6a',7',9',10'-hexahydro-5' $H$-spiro[indene-2,6'-pyrrolo[4,3,2-de][1,4]thiazino[4,3-a] quinoline]-1,3,5'-trione (3u)

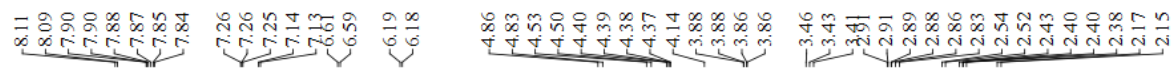
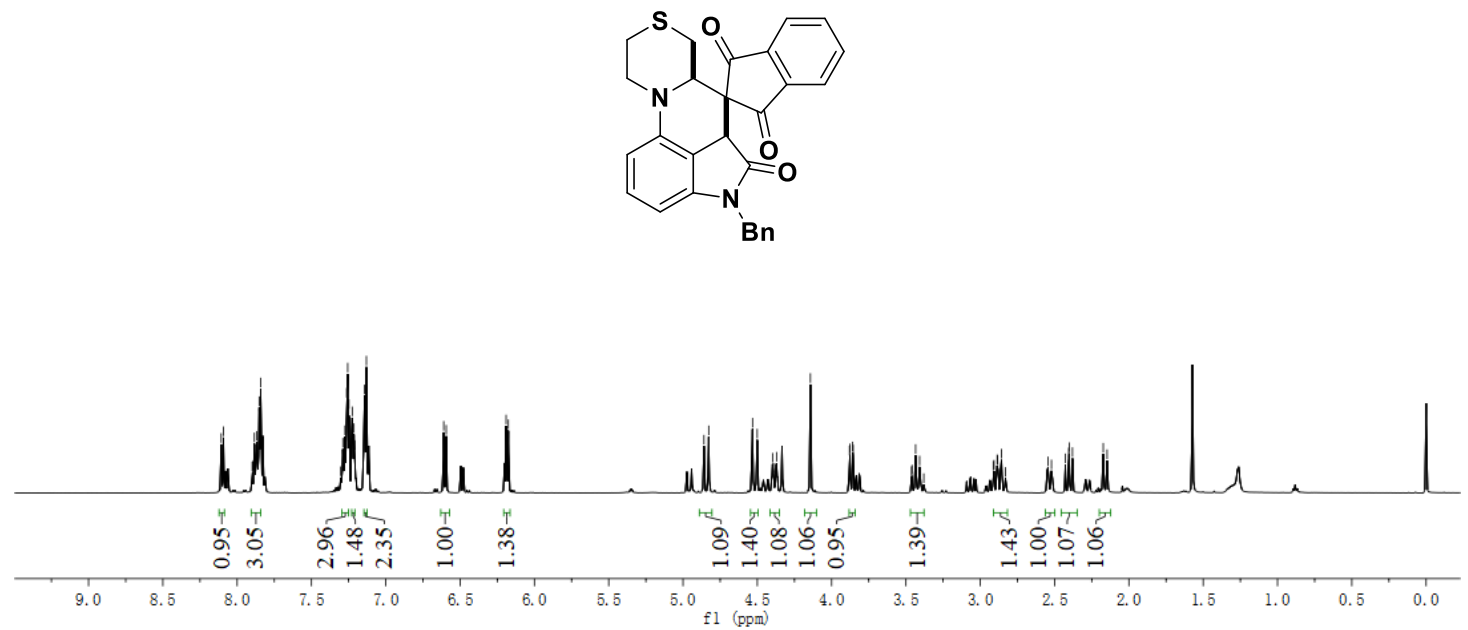

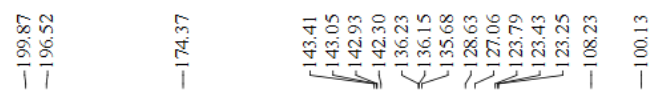

jịn
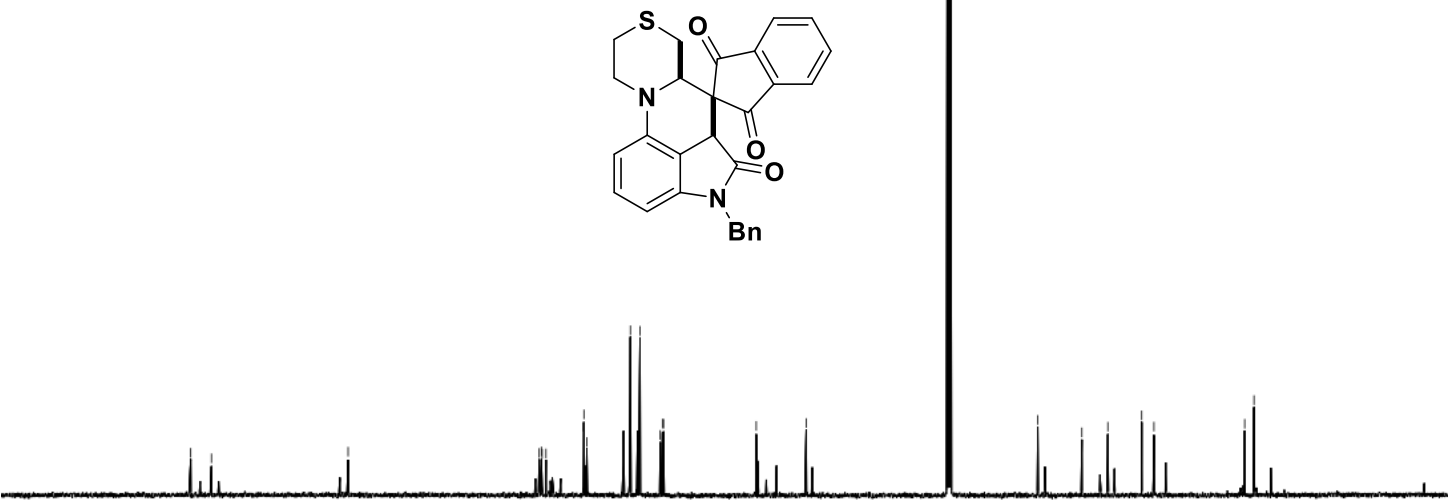

\begin{tabular}{lllllllllllllllllllllllllll}
\hline 30 & 220 & 210 & 200 & 190 & 180 & 170 & 160 & 150 & 140 & 130 & 120 & 110 & 100 & 90 & 80 & 70 & 60 & 50 & 40 & 30 & 20 & 10 & 0
\end{tabular} 

ene]-1',3',5-trione (3v)

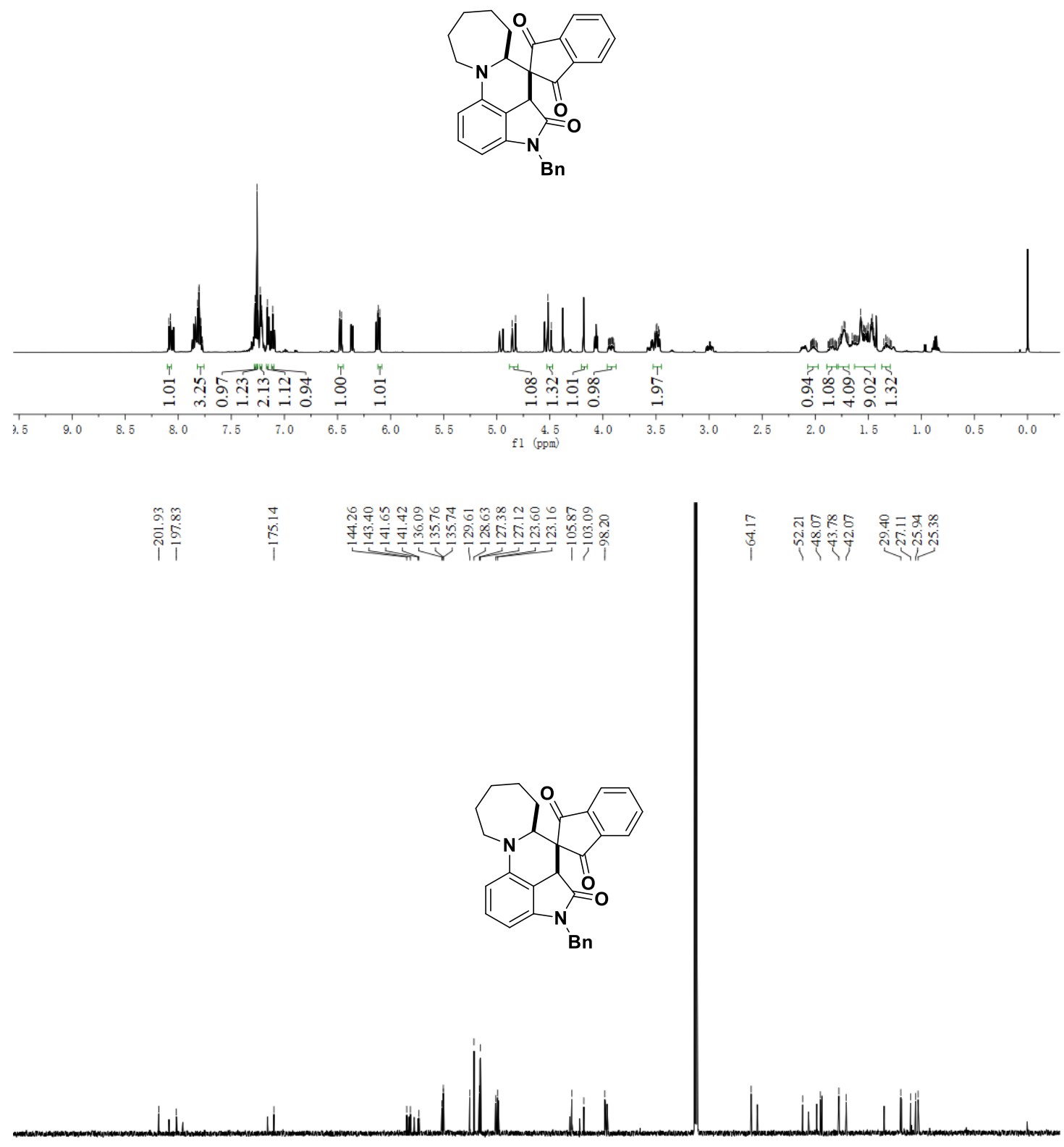

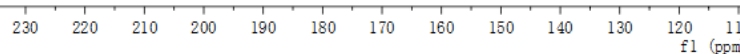


1'-benzyl-5'-methyl-4'-phenyl-1',2a',4',5'-tetrahydro-2' $H$-spiro[indene-2,3'-pyrrolo[4,3,2-de]quin oline]-1,2',3-trione (3w)

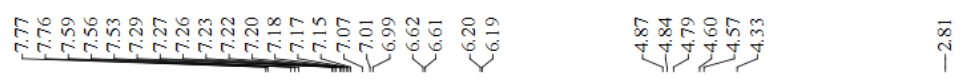
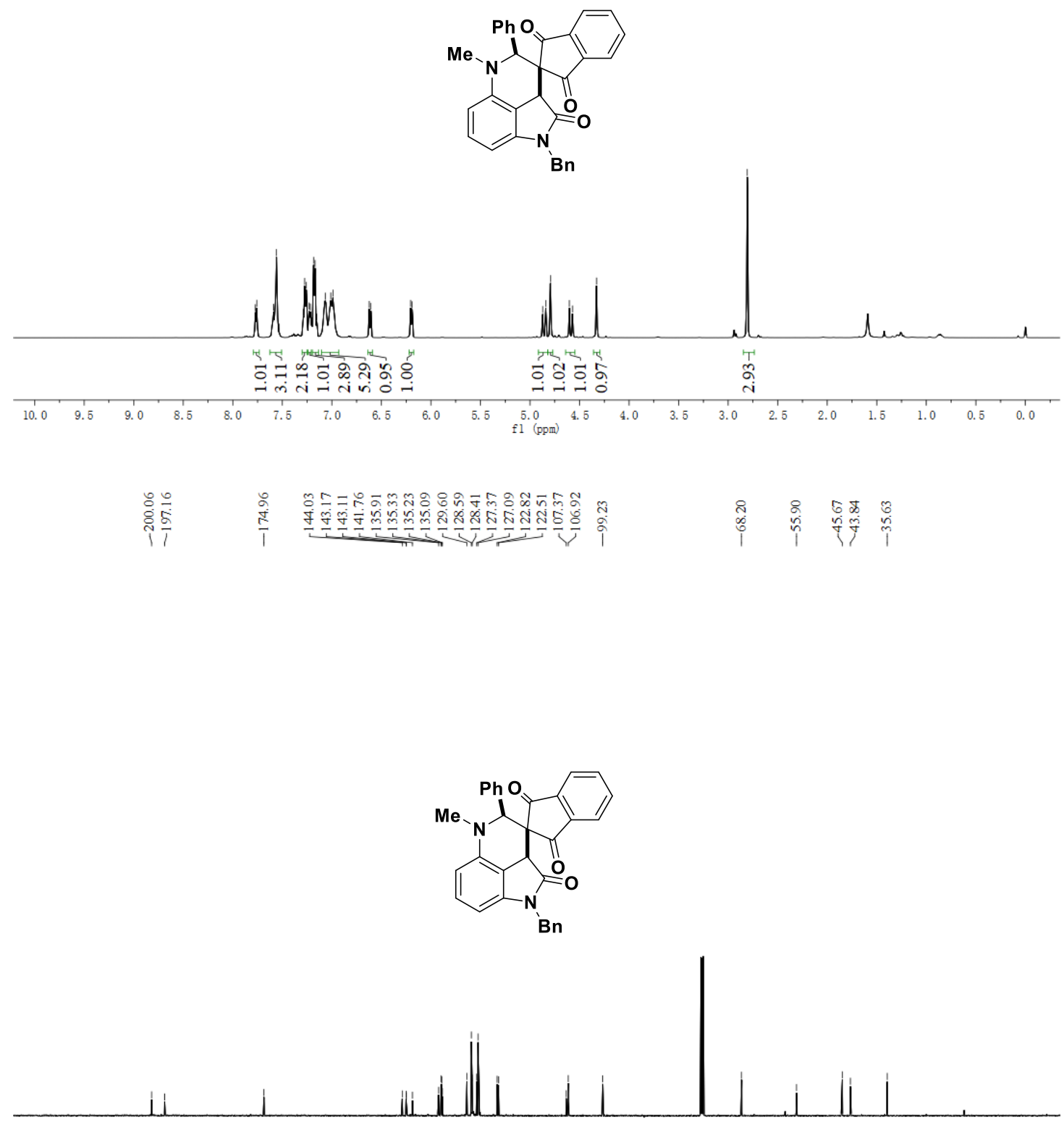

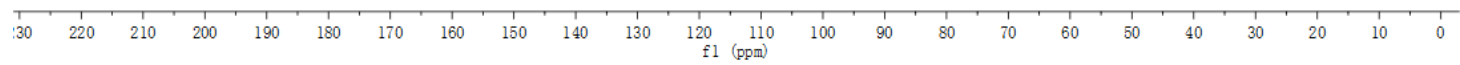


4-benzyl-1',3'-dimethyl-4,5a,6a,7,8,9-hexahydro-2'H,5H-spiro[dipyrrolo[1,2-a:4',3',2'-de]quinolin e-6,5'-pyrimidine]-2',4',5,6'(1'H,3'H)-tetraone (3x)

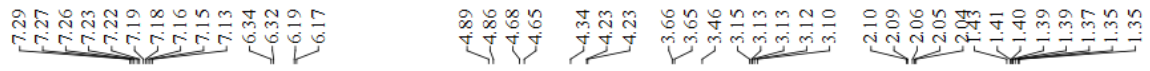
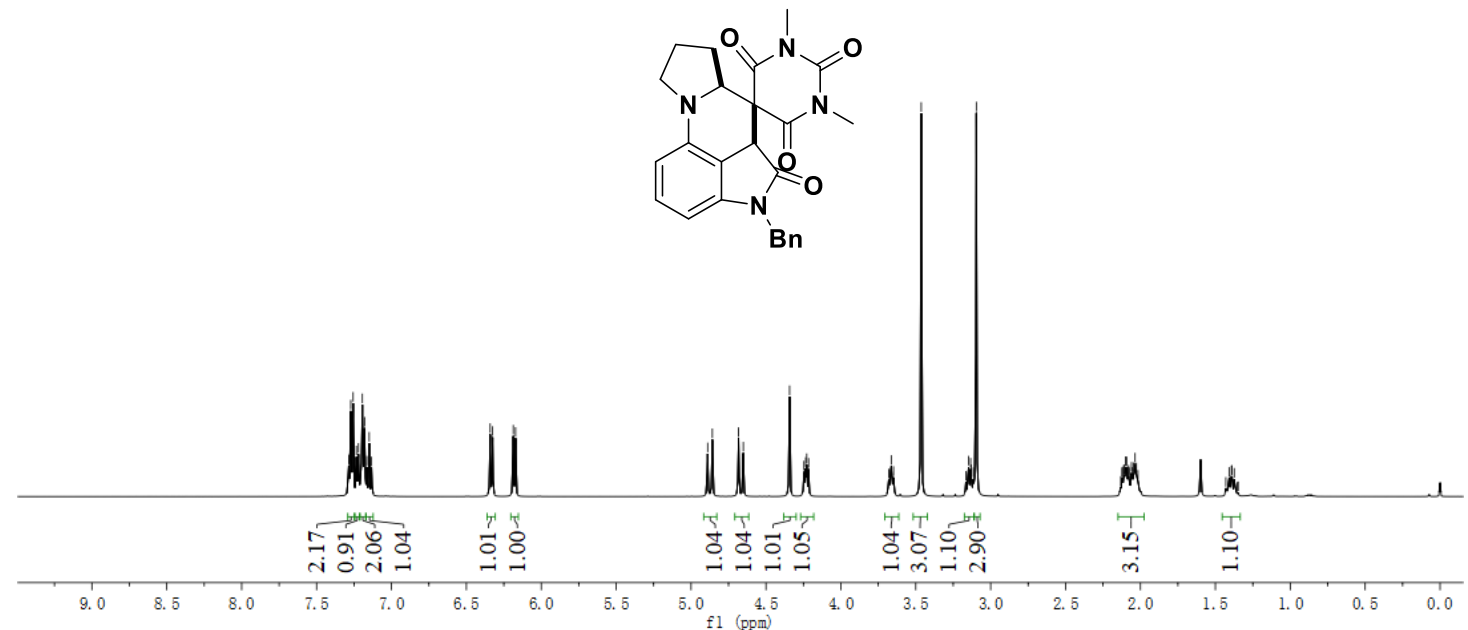

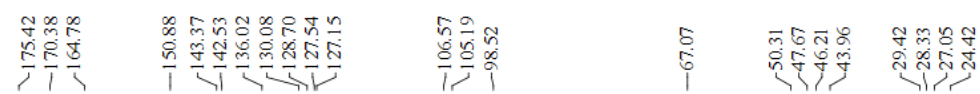
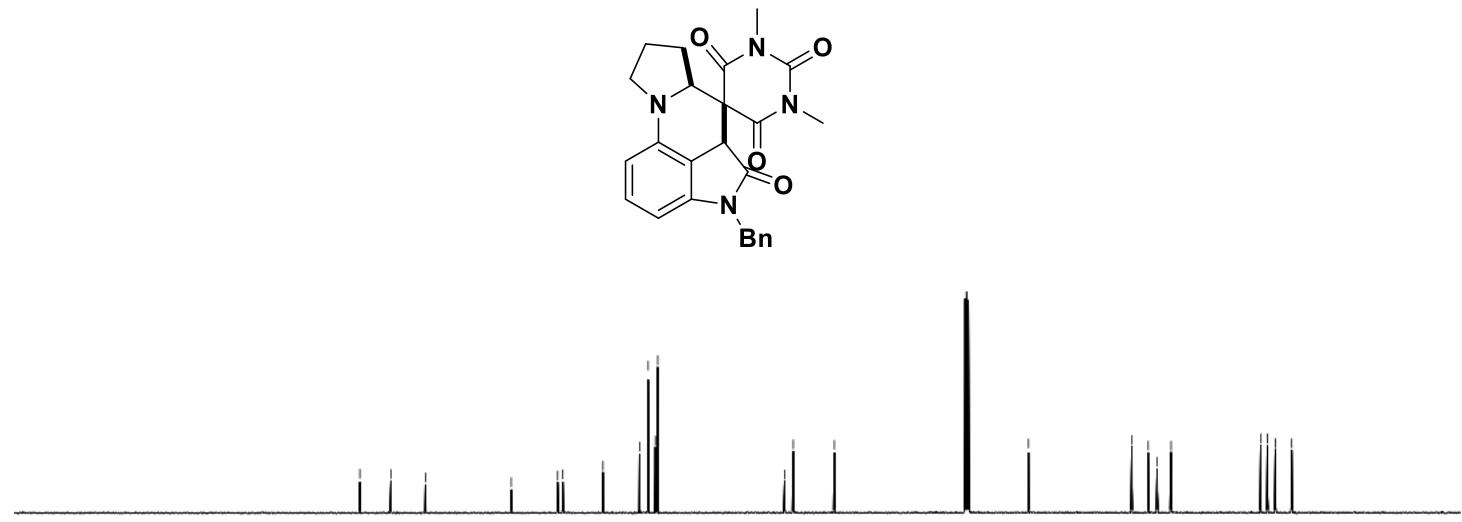

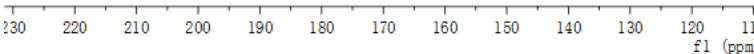

